\title{
Reversible lodine Capture by Nonporous Adaptive Crystals of a Bipyridine Cage
}

\author{
Dan Luo, ${ }^{a}$ Yanlei He, ${ }^{\mathrm{a}}$ Jinya Tian, ${ }^{\mathrm{a}}$ Jonathan L. Sessler, ${ }^{\mathrm{b},{ }^{*}}$ and Xiaodong Chi ${ }^{\mathrm{a},{ }^{*}}$ \\ ${ }^{a}$ State Key Laboratory of Materials Processing and Die \& Mould Technology, School of Materials \\ Science and Engineering, Huazhong University of Science and Technology, Wuhan 430074, China \\ ${ }^{\mathrm{b}}$ Department of Chemistry, The University of Texas at Austin, 105 E. 24th Street, Stop A5300, Austin, \\ Texas 78712-1224, USA.
}

\section{Supporting Information}

1. Materials and Methods $\quad$ S2

2. Synthesis and Characterization of BPy-Cage S3

3. X-ray Experimental Data for BPy-Cage $\quad S 4$

4. $\mathrm{N}_{2}$ Adsorption Isotherm for BPy-Cage S8

5. Powder X-ray Diffraction Study of BPy-Cage S8

6. Iodine Vapor Uptake Experiments $\quad S 9$

7. Iodine Capture Experiments in Solution $\quad S 12$

8. $\quad$ Iodine Release and Recyclability Studies of BPy-Cage S14

9. Studies of BPy-Cage-Modulated Acylation $\quad$ S17

10. Iodine Adsorption Capacities of Various Porous Adsorbents S18

11 Iodine Adsorption Capacities of 2,2'-BPy-DA S19

12 Studies of Bromine Capture from n-Hexane Solution S20

13. Supplementary References $\quad S 21$ 


\section{Materials and methods}

All chemicals and solvents were purchased from commercial suppliers and were used without further purification unless otherwise specified. Column chromatography was carried out on Merck GEDURAN Si60 (40-63 $\mu \mathrm{m})$. One-dimensional nuclear magnetic resonance (NMR) spectra were recorded on Bruker AVANCE 400 (400 MHz) spectrometers. The solvent signals were assigned by Fulmer et al. ${ }^{\mathrm{S} 1}$ All chemical shifts $(\delta)$ are quoted in ppm. The following abbreviations are used for convenience in reporting the multiplicity for NMR resonances: $\mathrm{s}=$ singlet, $\mathrm{d}=$ doublet, $\mathrm{t}=$ triplet, and $\mathrm{m}=$ multiplet. Data processing was performed with MestReNova software. High-resolution electrospray ionization mass spectrometry (ESI-MS) was performed on a micro TOF II instrument featuring a Z spray source with electrospray ionization and modular LockSpray interface. Powder X-ray diffraction (PXRD) data were collected on a Rigaku Ultimate-IV X-ray diffractometer operating at $40 \mathrm{kV} / 30 \mathrm{~mA}$ using the $\mathrm{Cu} \mathrm{K}_{\alpha}$ line $(\lambda=1.5418 \AA)$. Data were measured over the range of $5-50^{\circ}$ in $5^{\circ} / \mathrm{min}$ steps over 9 min. Thermogravimetric analyses (TGA) were carried out using a Q5000IR analyzer (TA Instruments) with an automated vertical overhead thermobalance. The samples were heated at $10{ }^{\circ} \mathrm{C} / \mathrm{min}$ using $\mathrm{N}_{2}$ as the protective gas at a flow rate of $50 \mathrm{ml} / \mathrm{min}$. Single crystal X-ray diffraction data were collected on a Rigaku XtaLAB PRO MM007HF X-ray diffractometer with graphite monochromated $\mathrm{Cu} \mathrm{K} \alpha$ radiation. Low-pressure gas adsorption measurements were performed on a Micrometritics Accelerated Surface Area and Porosimetry System (ASAP) 2020 surface area analyzer. Samples were degassed under dynamic vacuum for $12 \mathrm{~h}$ at $110^{\circ} \mathrm{C}$ prior to each measurement. $\mathrm{N}_{2}$ isotherms were measured using a liquid nitrogen bath $(77 \mathrm{~K})$. Scanning electron microscopy (SEM) and energy-dispersive spectroscopy (EDS) investigations were carried out on a JSM-5900LV instrument. UV-Vis spectra were recorded by using a JASCO V650 spectrometer. FTIR spectra were recorded as KBr pellets on a NEXUS 670 FTIR spectrometer. X-ray photoelectron spectroscopy (XPS) was carried out using a PHI5000 VersaProbe III instrument. 


\section{Synthesis and characterization of BPy-Cage}

Scheme S1. Synthesis of BPy-Cage<smiles>O=Cc1ccc(-c2ccc(C=O)cn2)nc1</smiles><smiles>CCC(CN)N(CCN)CCN</smiles>

$\mathrm{MeCN}$, rt.

\section{2,2'-BPy-DA}

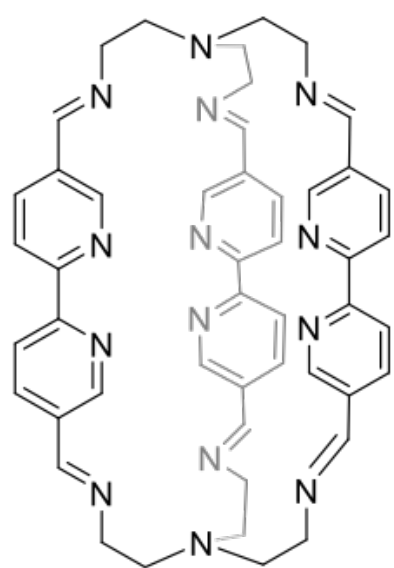

BPy-Cage

BPy-Cage: 2,2'-Bipyridyl-5,5'-dicarbaldehyde (2,2'-BPy-DA) (0.32 g, $1.51 \mathrm{mmol})$ was dissolved in $150 \mathrm{ml} \mathrm{CH}{ }_{3} \mathrm{CN}$. To the suspension, tris(2-aminoethyl)amine (Tren) ( $0.15 \mathrm{~mL}, 1.00$ mmol) in $5 \mathrm{ml} \mathrm{CH}_{3} \mathrm{CN}$ was added dropwise over $30 \mathrm{~min}$ at room temperature. The reaction mixture was stirred overnight at room temperature. A light-yellow precipitate was formed which was filtered and washed further with $\mathrm{MeCN}$. The filter cake was then dissolved in dichloromethane and filtered. The filtrate was concentrated to obtain the desired product BPyCage in $73 \%$ yield. ${ }^{1} \mathrm{H}$ NMR (400 MHz, $\left.\mathrm{CDCl}_{3}\right): \delta 8.44(\mathrm{~s}, 6 \mathrm{H}), 8.29(\mathrm{~s}, 6 \mathrm{H}), 7.98-7.96(\mathrm{~d}, J=$ $8.0 \mathrm{~Hz}, 6 \mathrm{H}), 7.80-7.77(\mathrm{dd}, J=12.0 \mathrm{~Hz}, J=4.0 \mathrm{~Hz}, 6 \mathrm{H}), 3.84(\mathrm{t}, 12 \mathrm{H}), 2.85(\mathrm{t}, 12 \mathrm{H}) ;{ }^{13} \mathrm{C} \mathrm{NMR}$ $\left(101 \mathrm{MHz}, \mathrm{CDCl}_{3}\right) \delta 158.06,155.29,148.99,132.67,129.82,120.11,57.08,51.43$. ESI-MS $\left(\mathrm{C}_{48} \mathrm{H}_{48} \mathrm{~N}_{14}\right): m / z$ 821.4301.

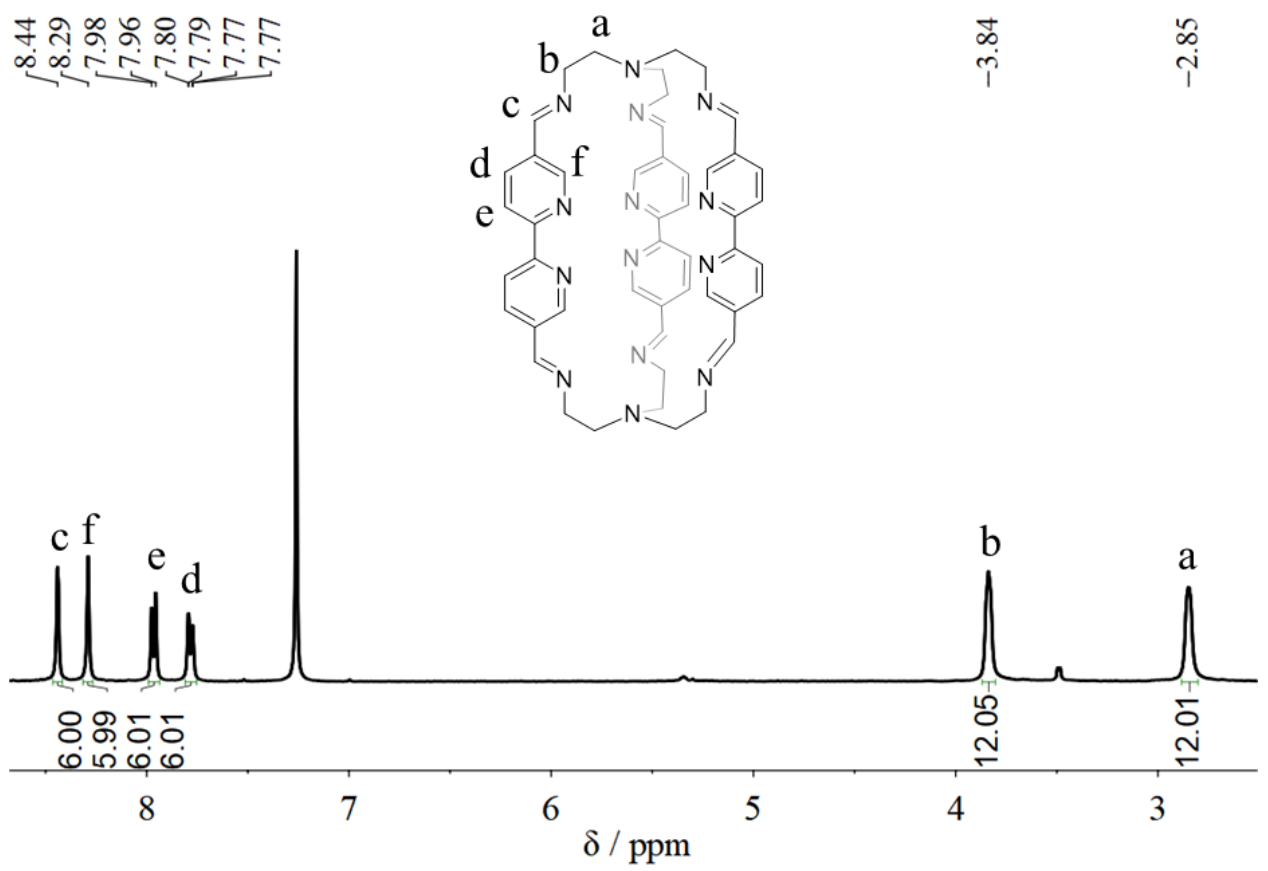

Figure S1. ${ }^{1} \mathrm{H}$ NMR spectrum (400 MHz, 298K, $\left.\mathrm{CDCl}_{3}\right)$ of BPy-Cage. 


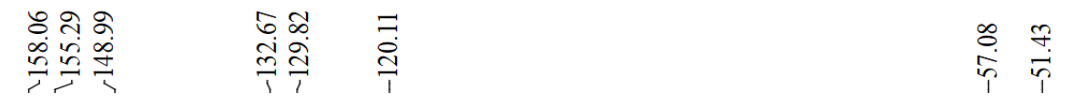

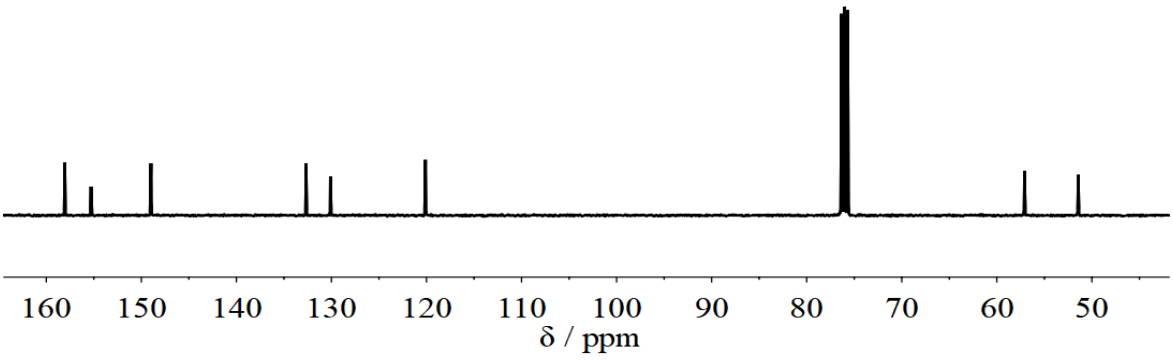

Figure S2. ${ }^{13} \mathrm{C}$ NMR spectrum $\left(101 \mathrm{MHz}, 298 \mathrm{~K}, \mathrm{CDCl}_{3}\right)$ of BPy-Cage.

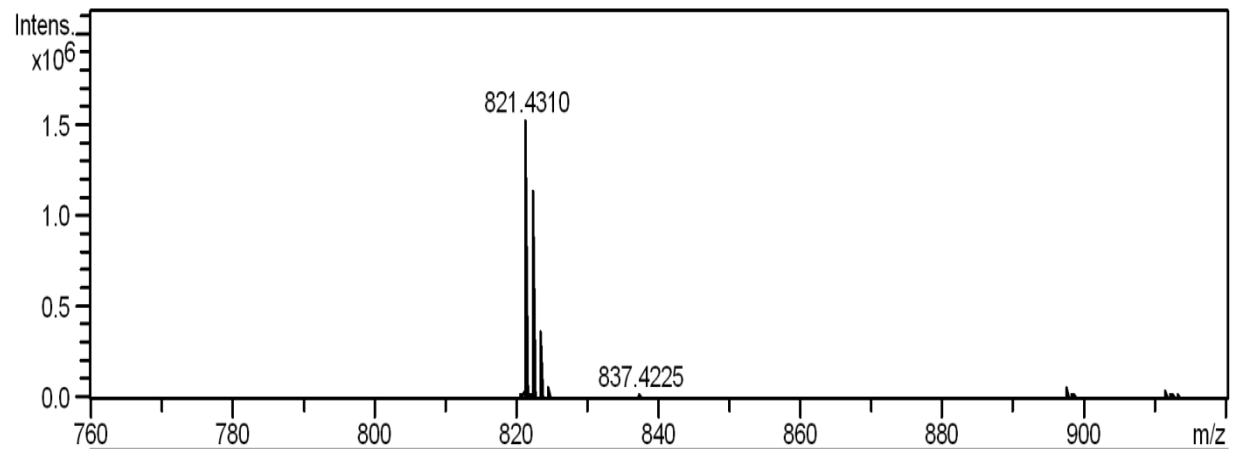

Figure S3. ESI-MS spectrum of BPy-Cage.

\section{X-ray experimental data for BPy-Cage}

X-ray experimental for $\mathrm{C}_{48} \mathrm{H}_{52} \mathrm{~N}_{14} \mathrm{O}_{2}$ : Crystals grew as colorless needle (2 $\mathbf{H}_{2} \mathbf{O} @$ BPy-Cage),

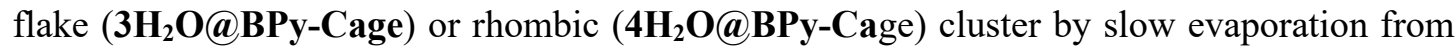
chloroform/hexane. The data crystal was cut from a cluster of crystals and had approximate dimensions; $0.2 \times 0.15 \times 0.03 \mathrm{~mm}$. The data were collected on XtaLAB PRO MM007HF Cu Rigaku, using a $\mu$-focus $\mathrm{Cu} \mathrm{K \alpha}$ radiation source $(\lambda=1.5418 \AA)$ with collimating mirror monochromators.

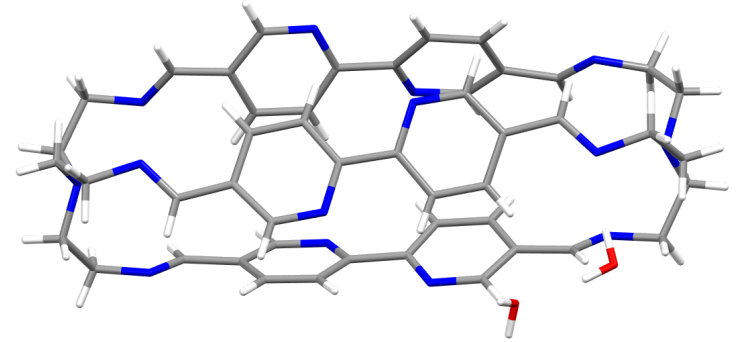

Figure S4. Single crystal structure of $\mathbf{2 H}_{2} \mathrm{O} @$ BPy-Cage. 
Table S1. Experimental single crystal X-ray data for $\mathbf{2} \mathbf{H}_{2} \mathbf{O} @ \mathbf{B P y}$-Cage (CCDC number: 2109890).

\begin{tabular}{|c|c|}
\hline Empirical formula & $\mathrm{C}_{48} \mathrm{H}_{52} \mathrm{~N}_{14} \mathrm{O}_{2}$ \\
\hline Formula weight & 857.03 \\
\hline Temperature/K & $100.00(10)$ \\
\hline Crystal system & monoclinic \\
\hline Space group & $\mathrm{P} 2{ }_{1} / \mathrm{n}$ \\
\hline $\mathrm{a} / \AA$ & $9.31914(9)$ \\
\hline $\mathrm{b} / \AA$ & $36.4432(3)$ \\
\hline $\mathrm{c} / \AA$ & $13.20189(11)$ \\
\hline$\alpha /{ }^{\circ}$ & 90 \\
\hline$\beta /{ }^{\circ}$ & 91.4663(9) \\
\hline$\gamma /{ }^{\circ}$ & 90 \\
\hline Volume $/ \AA^{3}$ & $4482.15(7)$ \\
\hline $\mathrm{Z}$ & 4 \\
\hline$\rho_{\text {calc }} \mathrm{g} / \mathrm{cm}^{3}$ & 1.270 \\
\hline$\mu / \mathrm{mm}^{-1}$ & 0.659 \\
\hline $\mathrm{F}(000)$ & 1816.0 \\
\hline Crystal size $/ \mathrm{mm}^{3}$ & $0.4 \times 0.2 \times 0.03$ \\
\hline Radiation & $\mathrm{CuK} \alpha(\lambda=1.54184)$ \\
\hline $2 \theta$ range for data collection ${ }^{\circ}$ & 7.124 to 147.604 \\
\hline Index ranges & $-11 \leq \mathrm{h} \leq 6,-44 \leq \mathrm{k} \leq 44,-16 \leq 1 \leq 15$ \\
\hline Reflections collected & 48449 \\
\hline Independent reflections & $8984\left[\mathrm{R}_{\text {int }}=0.0423, \mathrm{R}_{\text {sigma }}=0.0284\right]$ \\
\hline Data/restraints/parameters & $8984 / 0 / 584$ \\
\hline Goodness-of-fit on $\mathrm{F}^{2}$ & 1.046 \\
\hline Final $R$ indexes $[\mathrm{I}>=2 \sigma(\mathrm{I})]$ & $\mathrm{R}_{1}=0.0410, \mathrm{wR}_{2}=0.1013$ \\
\hline Final R indexes [all data] & $\mathrm{R}_{1}=0.0474, \mathrm{wR}_{2}=0.1049$ \\
\hline
\end{tabular}

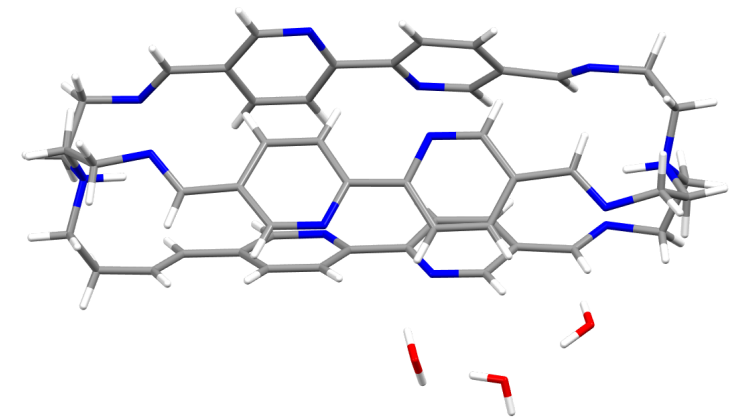

Figure S5. Single crystal structure of $3 \mathrm{H}_{2} \mathrm{O} @ \mathbf{B P y}-$ Cage. 
Table S2. Experimental single crystal X-ray data for $\mathbf{3} \mathbf{H}_{2} \mathbf{O} @ \mathbf{B P y}-\mathbf{C a g e}(\mathrm{CCDC}$ number: 2109894).

\begin{tabular}{|c|c|}
\hline Empirical formula & $\mathrm{C}_{48} \mathrm{H}_{54} \mathrm{~N}_{14} \mathrm{O}_{3}$ \\
\hline Formula weight & 875.04 \\
\hline Temperature/K & $99.99(10)$ \\
\hline Crystal system & triclinic \\
\hline Space group & $\mathrm{P}-1$ \\
\hline $\mathrm{a} / \AA$ & $11.8481(4)$ \\
\hline $\mathrm{b} / \AA$ & $12.8189(5)$ \\
\hline $\mathrm{c} / \AA$ & $15.4468(4)$ \\
\hline$\alpha /^{\circ}$ & $89.593(3)$ \\
\hline$\beta /{ }^{\circ}$ & $77.728(2)$ \\
\hline$\gamma /{ }^{\circ}$ & $81.339(3)$ \\
\hline Volume $/ \AA^{3}$ & $2265.55(13)$ \\
\hline $\mathrm{Z}$ & 2 \\
\hline$\rho_{\text {calc }} \mathrm{g} / \mathrm{cm}^{3}$ & 1.283 \\
\hline$\mu / \mathrm{mm}^{-1}$ & 0.678 \\
\hline $\mathrm{F}(000)$ & 928.0 \\
\hline Crystal size $/ \mathrm{mm}^{3}$ & $0.4 \times 0.2 \times 0.03$ \\
\hline Radiation & $\mathrm{CuK} \alpha(\lambda=1.54184)$ \\
\hline $2 \theta$ range for data collection ${ }^{\circ}$ & 5.858 to 103.388 \\
\hline Index ranges & $-11 \leq \mathrm{h} \leq 12,-12 \leq \mathrm{k} \leq 13,-15 \leq 1 \leq 15$ \\
\hline Reflections collected & 24971 \\
\hline Independent reflections & $4924\left[\mathrm{R}_{\text {int }}=0.0684, \mathrm{R}_{\text {sigma }}=0.0421\right]$ \\
\hline Data/restraints/parameters & $4924 / 2 / 585$ \\
\hline Goodness-of-fit on $\mathrm{F}^{2}$ & 1.042 \\
\hline Final $\mathrm{R}$ indexes $[\mathrm{I}>=2 \sigma(\mathrm{I})]$ & $\mathrm{R}_{1}=0.0669, \mathrm{wR}_{2}=0.1926$ \\
\hline Final $\mathrm{R}$ indexes [all data] & $\mathrm{R}_{1}=0.0796, \mathrm{wR}_{2}=0.2019$ \\
\hline
\end{tabular}

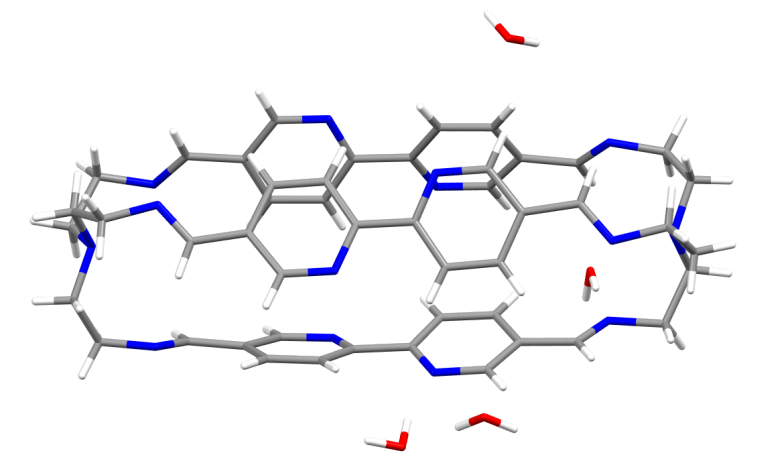

Figure S6. Single crystal structure of $\mathbf{4} \mathbf{H}_{2} \mathrm{O} @$ BPy-Cage. 
Table S3. Experimental single crystal X-ray data for $\mathbf{4} \mathbf{H}_{2} \mathbf{O} @ \mathbf{B P y}$-Cage (CCDC number: 2109887).

\begin{tabular}{|c|c|}
\hline Empirical formula & $\mathrm{C}_{48} \mathrm{H}_{56} \mathrm{~N}_{14} \mathrm{O}_{4}$ \\
\hline Formula weight & 893.06 \\
\hline Temperature/K & 100 \\
\hline Crystal system & monoclinic \\
\hline Space group & $\mathrm{P} 2{ }_{1} / \mathrm{c}$ \\
\hline $\mathrm{a} / \AA ̊$ & $16.9342(2)$ \\
\hline $\mathrm{b} / \AA$ & $16.3055(2)$ \\
\hline $\mathrm{c} / \AA$ & $17.0474(2)$ \\
\hline$\alpha /^{\circ}$ & 90 \\
\hline$\beta /{ }^{\circ}$ & $98.0560(10)$ \\
\hline$\gamma /{ }^{\circ}$ & 90 \\
\hline Volume $/ \AA^{3}$ & $4660.69(10)$ \\
\hline $\mathrm{Z}$ & 4 \\
\hline$\rho_{\text {calc }} \mathrm{g} / \mathrm{cm}^{3}$ & 1.273 \\
\hline$\mu / \mathrm{mm}^{-1}$ & 0.686 \\
\hline $\mathrm{F}(000)$ & 1896.0 \\
\hline Crystal size $/ \mathrm{mm}^{3}$ & $0.15 \times 0.08 \times 0.05$ \\
\hline Radiation & $\mathrm{CuK} \alpha(\lambda=1.54184)$ \\
\hline $2 \theta$ range for data collection $/{ }^{\circ}$ & 5.27 to 148.246 \\
\hline Index ranges & $-20 \leq \mathrm{h} \leq 16,-20 \leq \mathrm{k} \leq 19,-20 \leq 1 \leq 21$ \\
\hline Reflections collected & 54284 \\
\hline Independent reflections & $9321\left[\mathrm{R}_{\text {int }}=0.0504, \mathrm{R}_{\text {sigma }}=0.0323\right]$ \\
\hline Data/restraints/parameters & $9321 / 0 / 628$ \\
\hline Goodness-of-fit on $\mathrm{F}^{2}$ & 1.038 \\
\hline Final $\mathrm{R}$ indexes $[\mathrm{I}>=2 \sigma(\mathrm{I})]$ & $\mathrm{R}_{1}=0.0407, \mathrm{wR}_{2}=0.1024$ \\
\hline Final $\mathrm{R}$ indexes [all data] & $\mathrm{R}_{1}=0.0488, \mathrm{wR}_{2}=0.1072$ \\
\hline
\end{tabular}


4. $\mathbf{N}_{2}$ adsorption isotherm for BPy-Cage

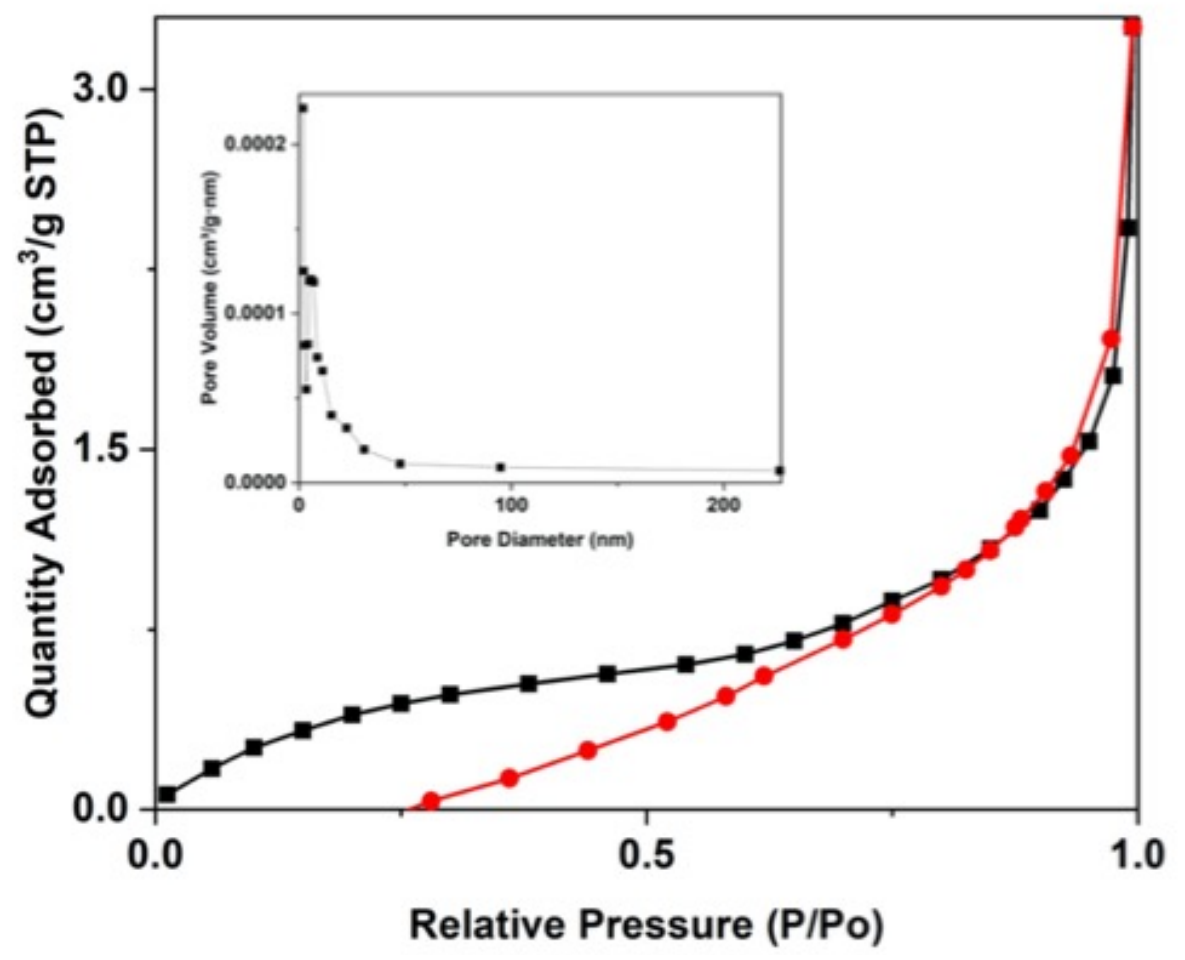

Figure S7. $\mathrm{N}_{2}$ adsorption isotherm for BPy-Cage. The BET surface area value is $1.8 \mathrm{~m}^{2} / \mathrm{g}$.

5. Powder X-ray diffraction patterns of BPy-Cage

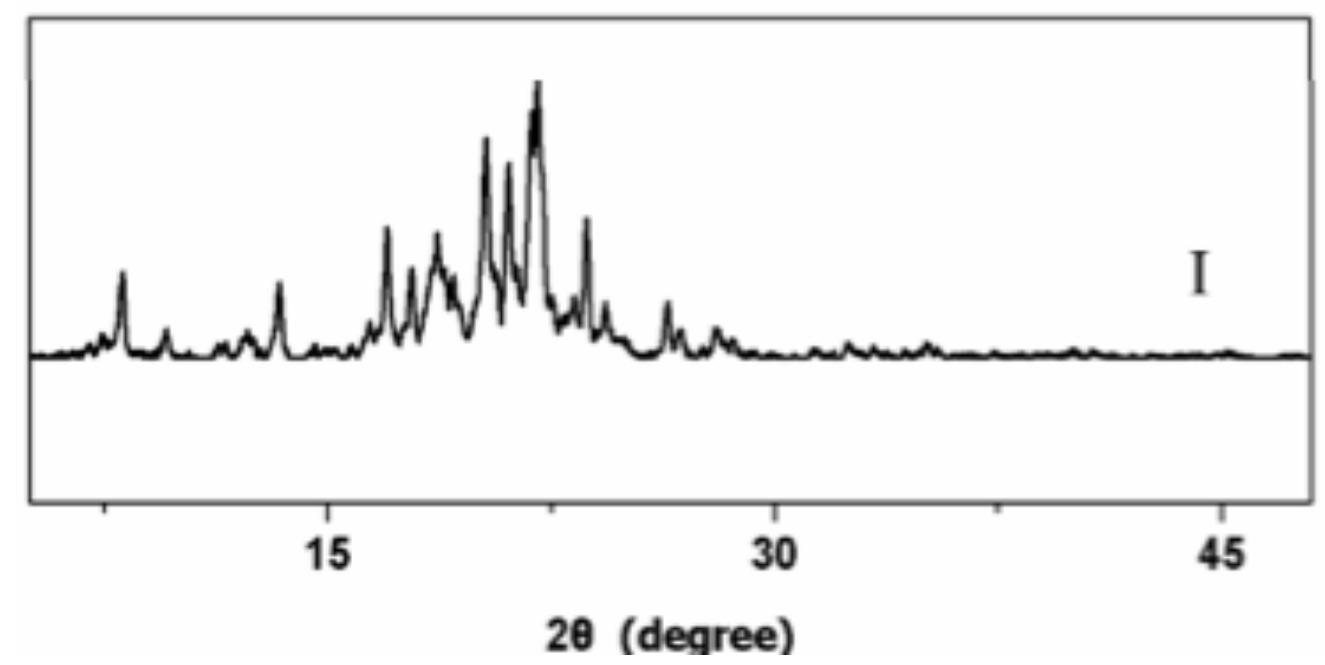

Figure S8. Powder X-ray diffraction patterns of BPy-Cage 


\section{Iodine Vapor Uptake Experiments}

Time-dependent iodine vapor uptake experiments were performed in the following procedure: $25 \mathrm{mg}$ of BPy-Cage in an open thermoresistant plastic cap $(30 \mathrm{~mL})$ and excessive of iodine was placed in a sealed glass vial $(100 \mathrm{~mL})$ and heated at $348 \mathrm{~K}$. After adsorption of the iodine vapor (0-24 h), the adsorbed BPy-Cage powder was cooled down to room temperature and analyzed by analytical balance, IR, TGA and PXRD.

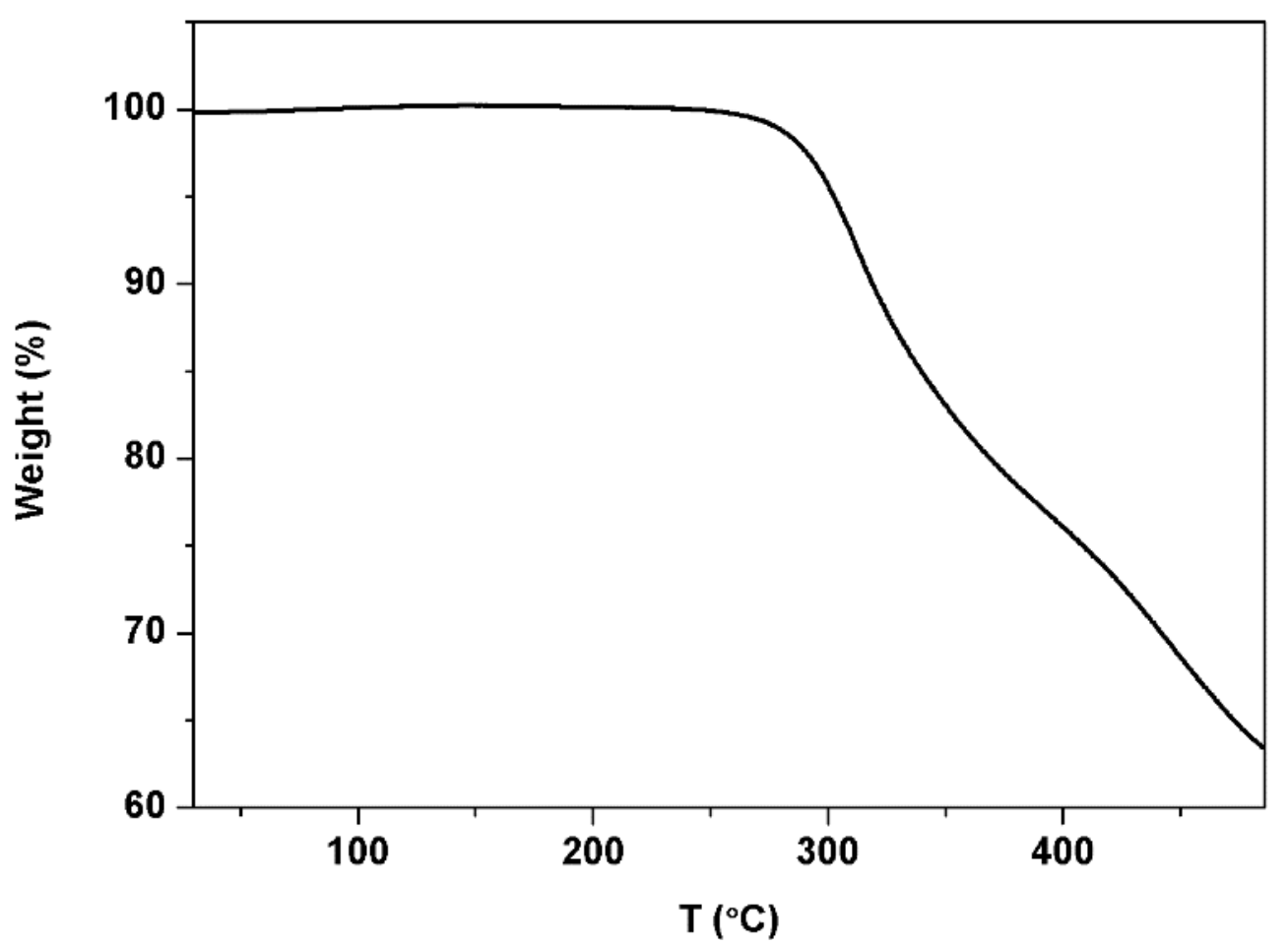

Figure S9. Thermogravimetric analysis of BPy-Cage. 


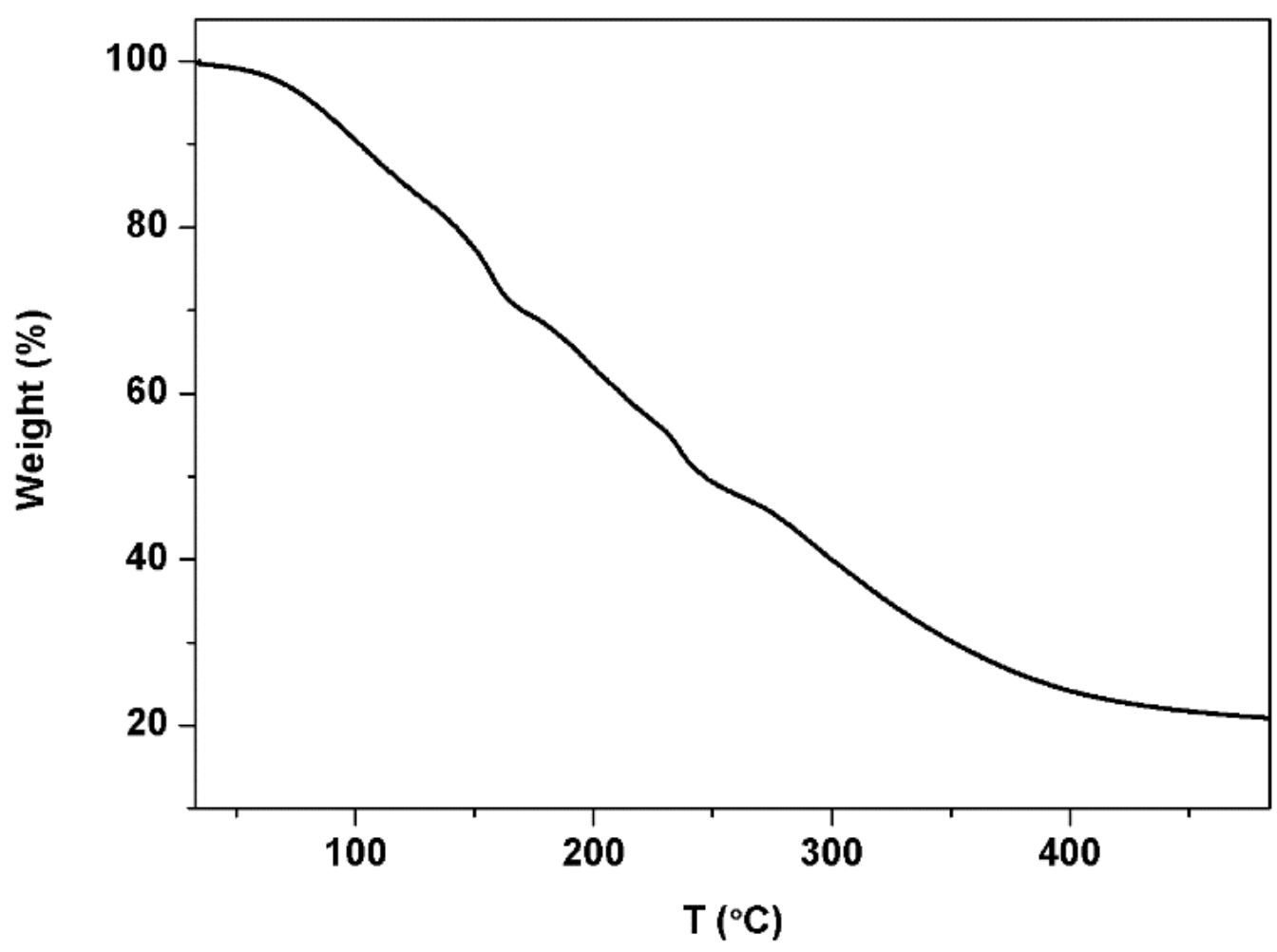

Figure S9. Thermogravimetric analysis of $\mathbf{I}_{2} @ \mathbf{B P y}$-Cage.

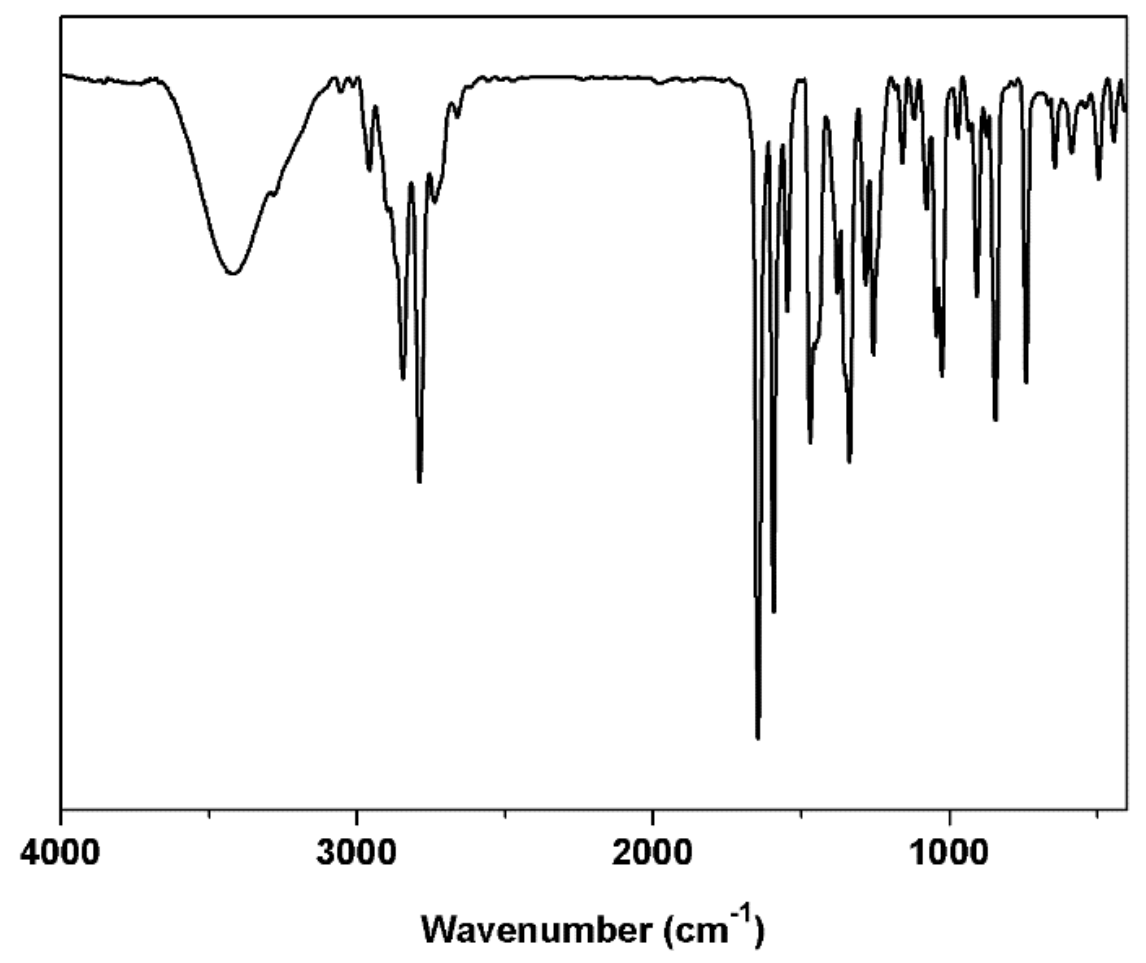

Figure S10. FT-IR spectra of BPy-Cage. 


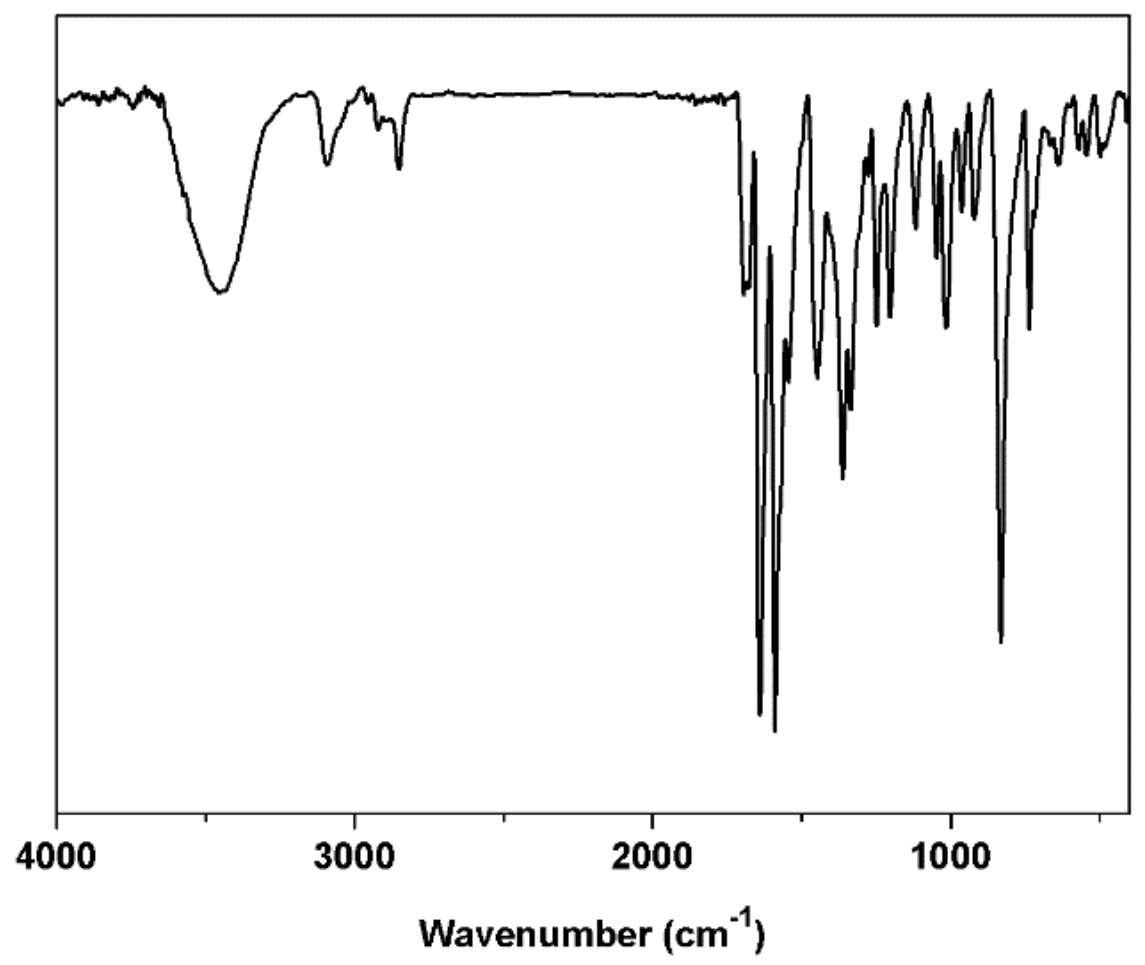

Figure S11. FT-IR spectra of $\mathbf{I}_{2} @$ BPy-Cage.

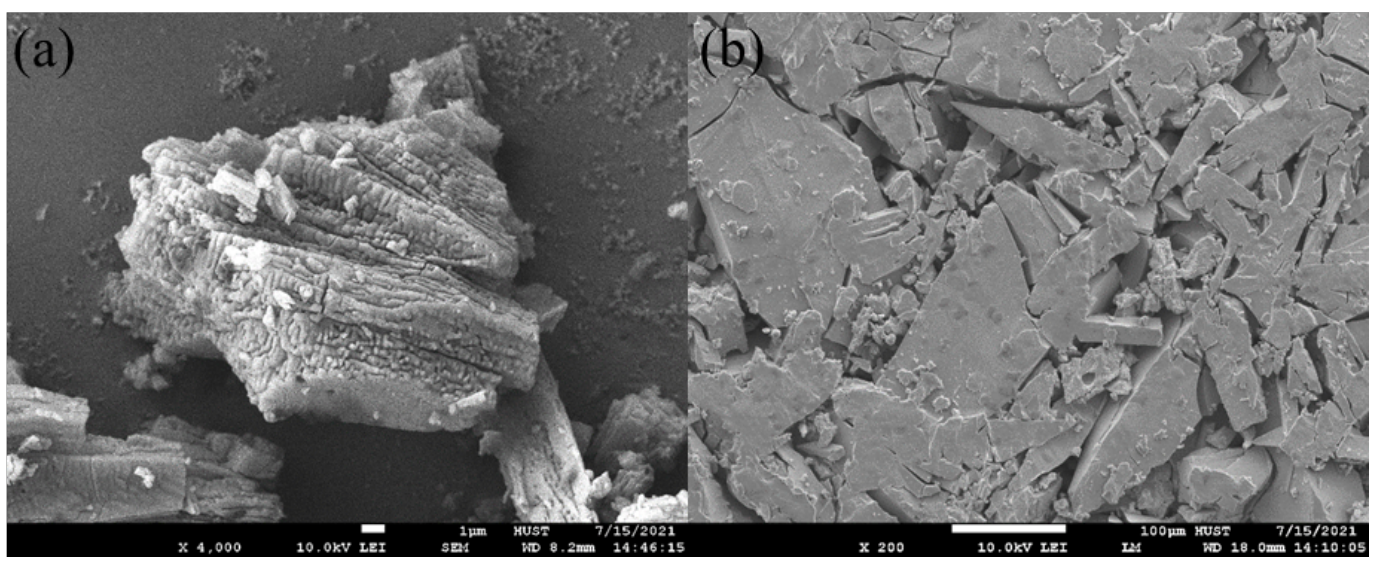

Figure S12. Scanning electron microscopy (SEM) image of (a) BPy-Cage and (b) BPy-Cage exposure to iodine vapor for $24 \mathrm{~h}$. 


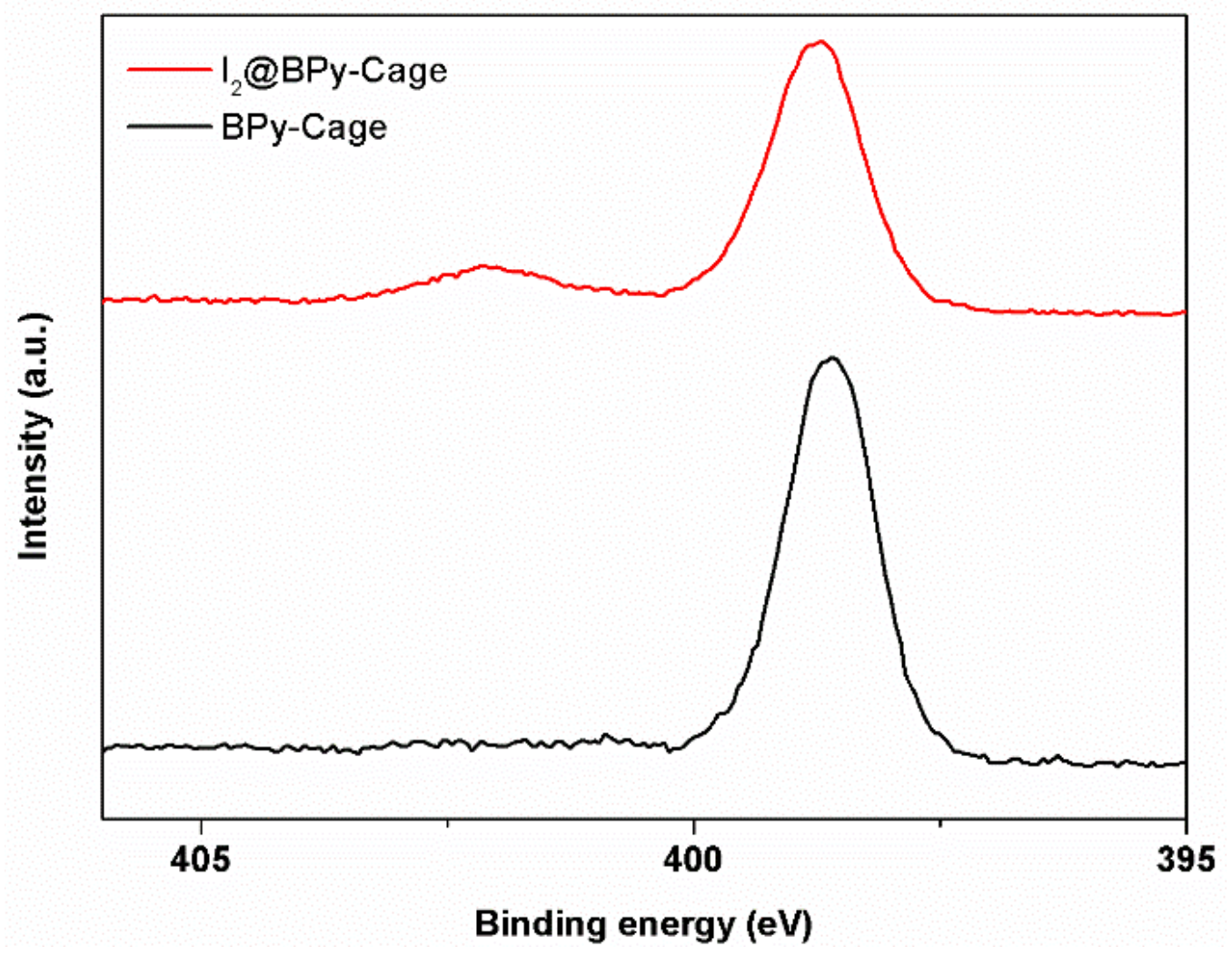

Figure S13. XPS spectra of N 1s for BPy-Cage and $\mathbf{I}_{2} @$ BPy-Cage.

\section{Iodine Capture Experiments in Solution}
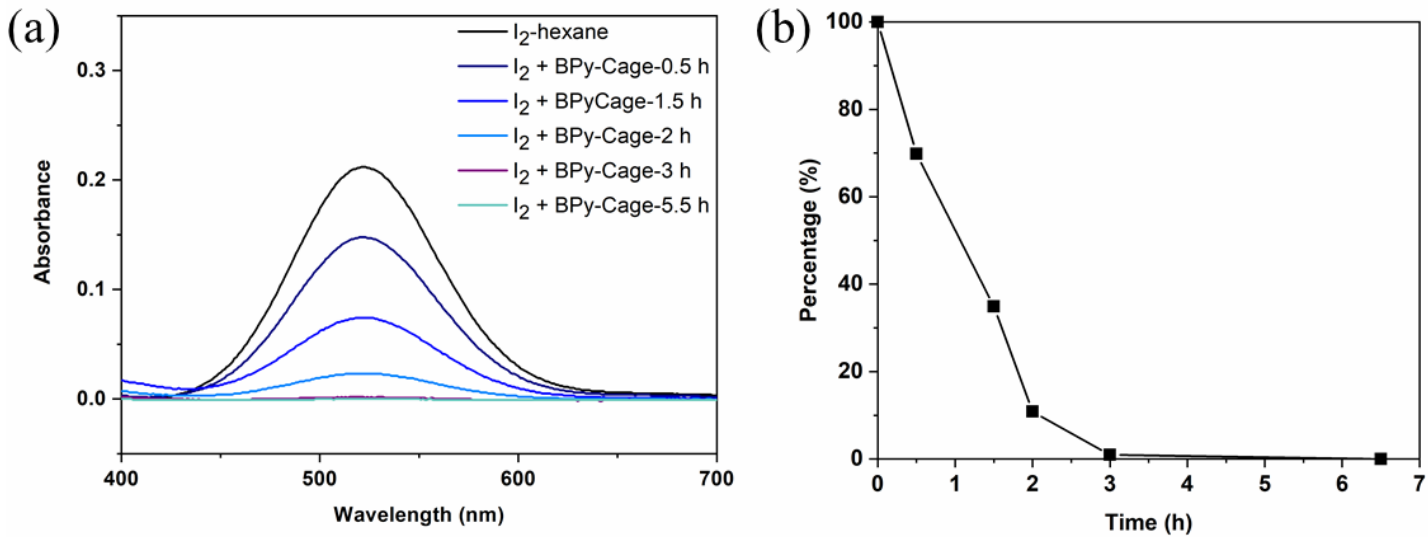

Figure S14. (a) Time-dependent UV/vis absorption spectra of an iodine/n-hexane solution upon the addition of BPy-Cage solid; (b) the iodine content in the n-hexane solution. 


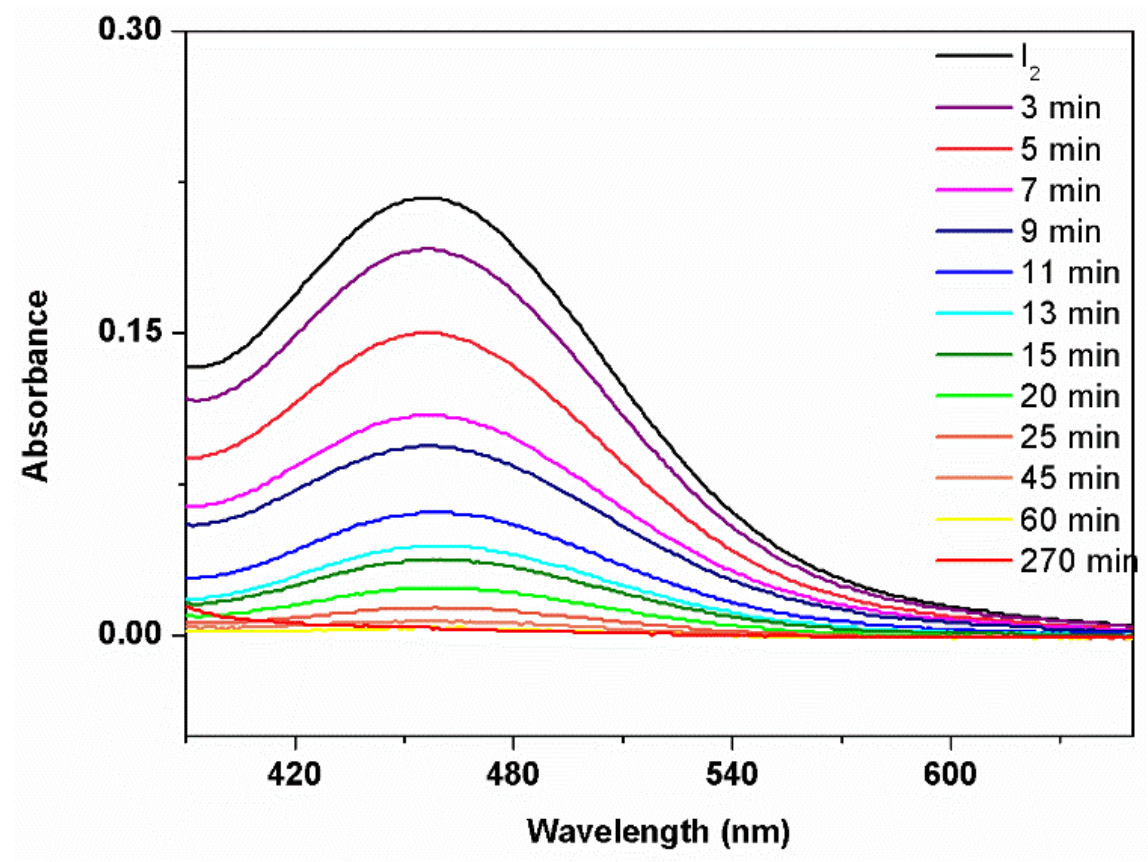

Figure S15. Time-dependent UV/vis absorption spectra of the iodine aqueous solution upon addition of BPy-Cage.

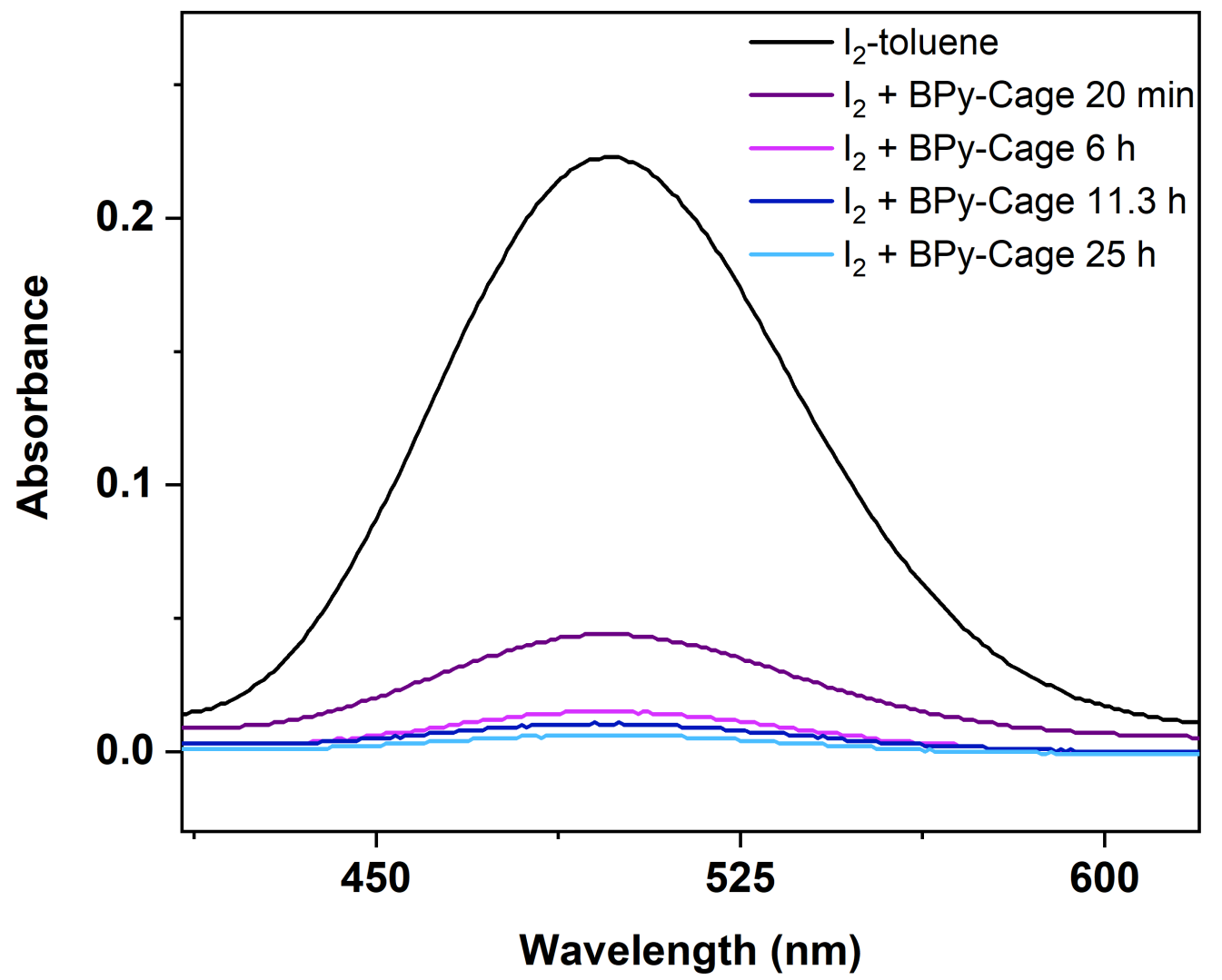

Figure S16. Time-dependent UV/vis absorption spectra of the iodine/toluene solution upon addition of BPy-Cage. 


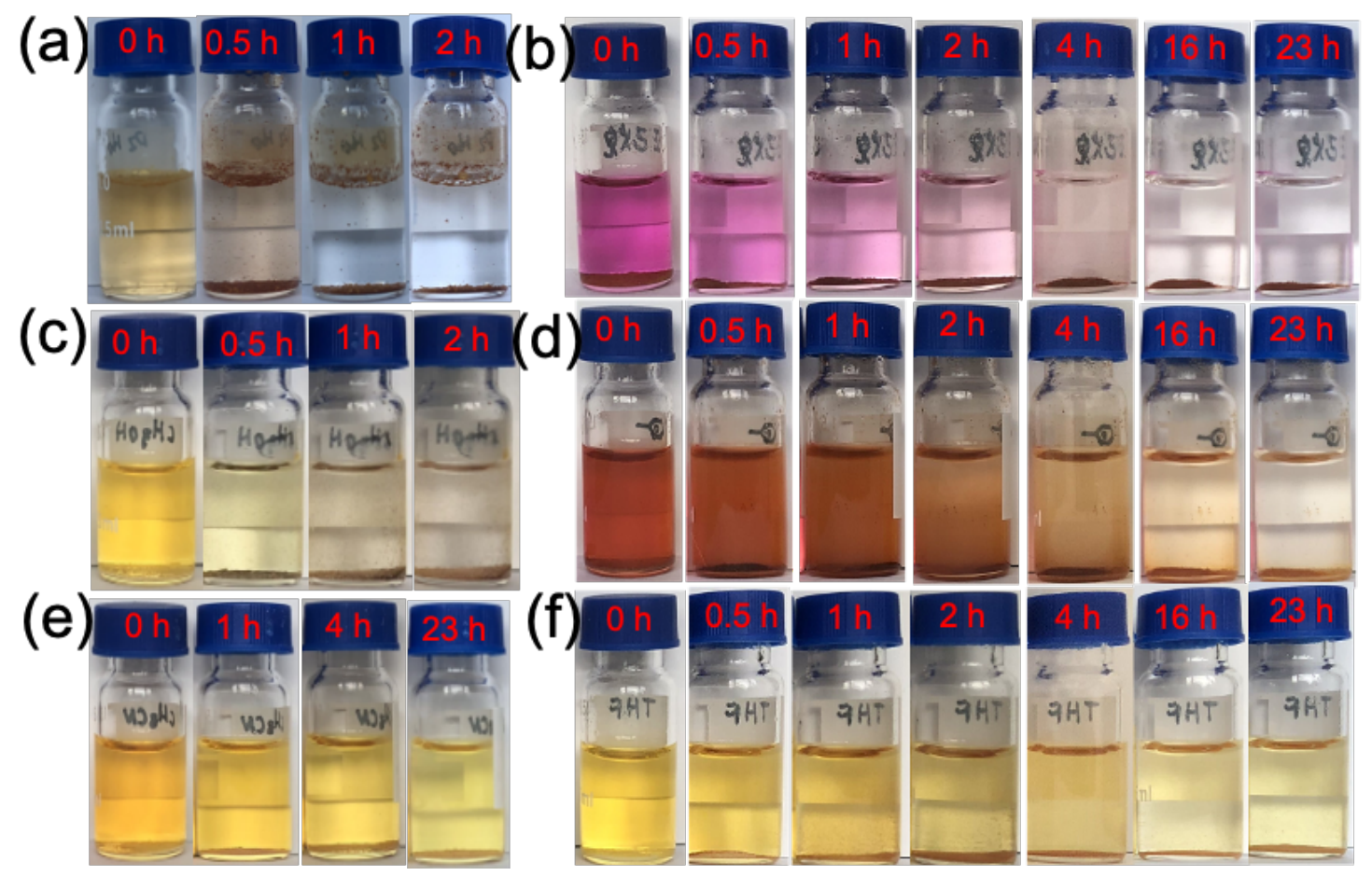

Figure S17. Time-dependent color change of the various iodine solution $(1.0 \mathrm{mM}, 3.0 \mathrm{~mL})$ upon addition of BPy-Cage (3.0 mg), iodine solution of (a) water, (b) n-hexane (c) methanol, (d) toluene, (e) acetonitrile, (f) tetrahydrofuran.

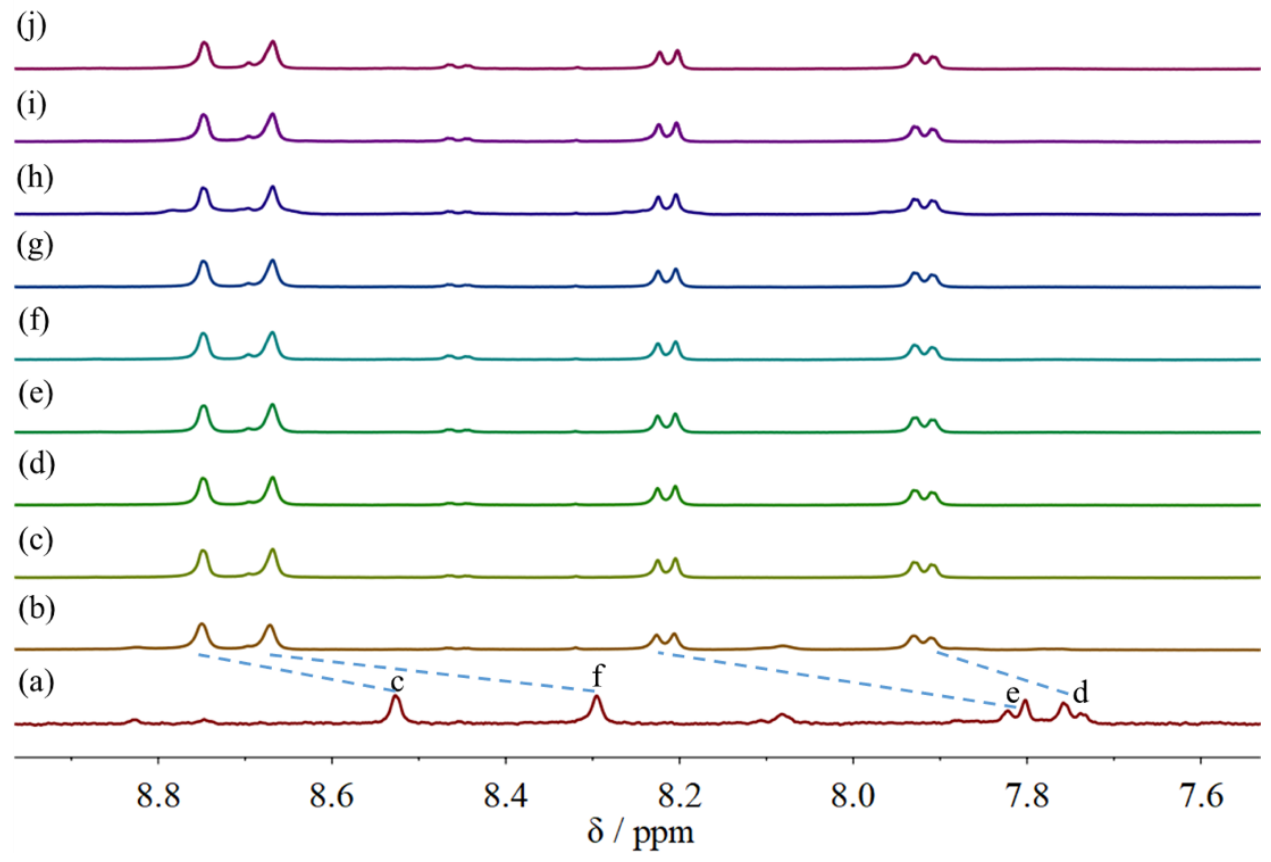

Figure S18. ${ }^{1} \mathrm{H}$ NMR spectra (400 MHz, DMSO- $d_{6}, 298 \mathrm{~K}$ ) of (a) BPy-Cage; (b) BPy-Cage +1 equiv $\mathrm{I}_{2}$; (c) BPy-Cage +3 equiv $\mathrm{I}_{2}$; (d) BPy-Cage +4 equiv $\mathrm{I}_{2}$; (e) BPy-Cage +5 equiv $\mathrm{I}_{2}$; (f) BPy-Cage +6 equiv $\mathrm{I}_{2}$; (g) BPy-Cage +7 equiv $\mathrm{I}_{2}$; (h) BPy-Cage +8 equiv $\mathrm{I}_{2}$; (i) BPy-Cage +10 equiv $\mathrm{I}_{2}$; (j) BPy-Cage +15 equiv $\mathrm{I}_{2}$.

\section{Iodine release and recyclability of BPy-Cage}


Iodine release induced in DMF solution: $\mathbf{I}_{\mathbf{2}} @ \mathbf{B P y}$-Cage was immersed in DMF to release the adsorbed iodine. When the release was deemed essentially complete, the resulting light yellow solid was recycled and analyzed by PXRD and IR spectrsocopy.

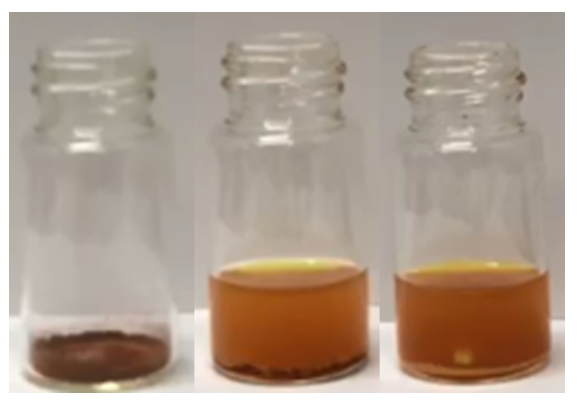

Figure S19. Photographs showing a color change when $3 \mathrm{mg}$ of $\mathbf{I}_{2} @ \mathbf{B P y}$-Cage solid was immersed in DMF solution. The colour of the solid changed from dark brown to light yellow because of the release of the iodine from the cage.

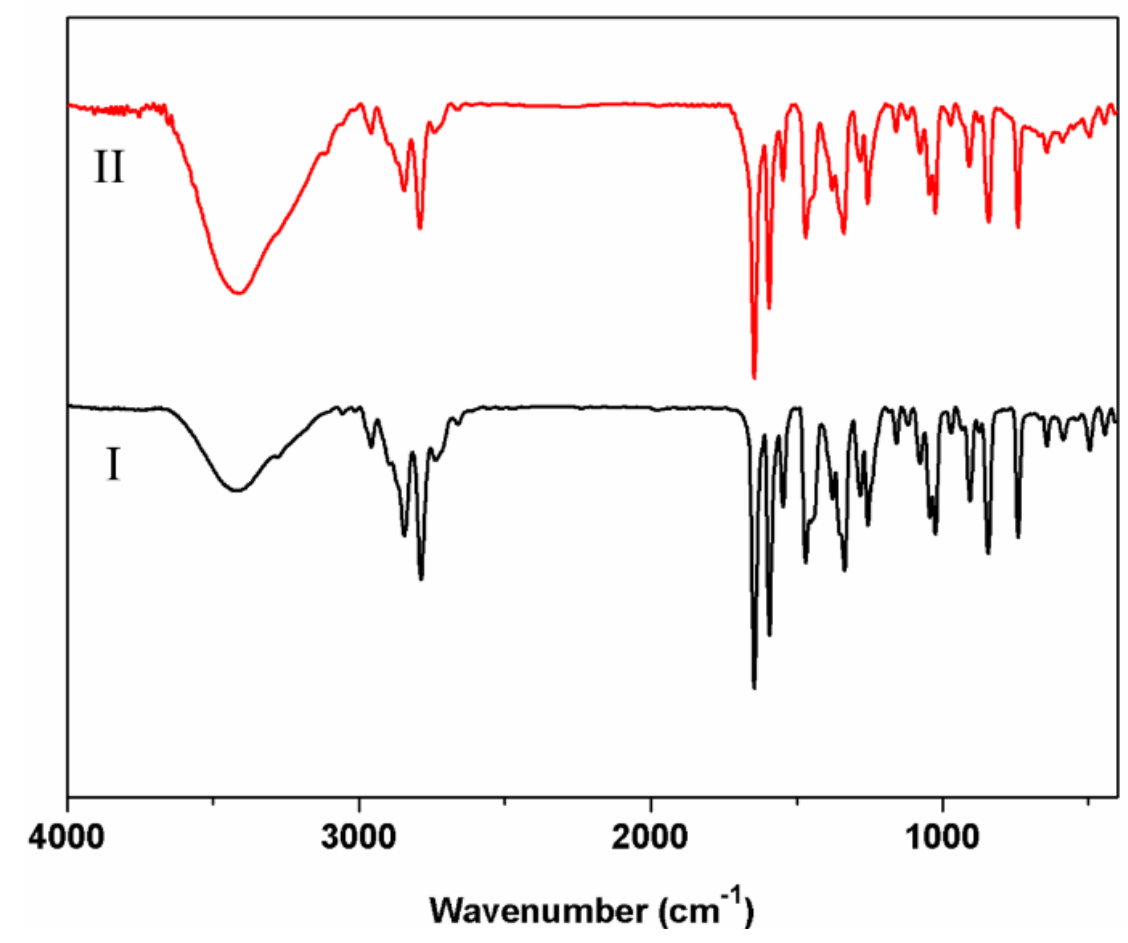

Figure S20. FT-IR spectra of BPy-Cage (I) and BPy-Cage after release of iodine (II). 


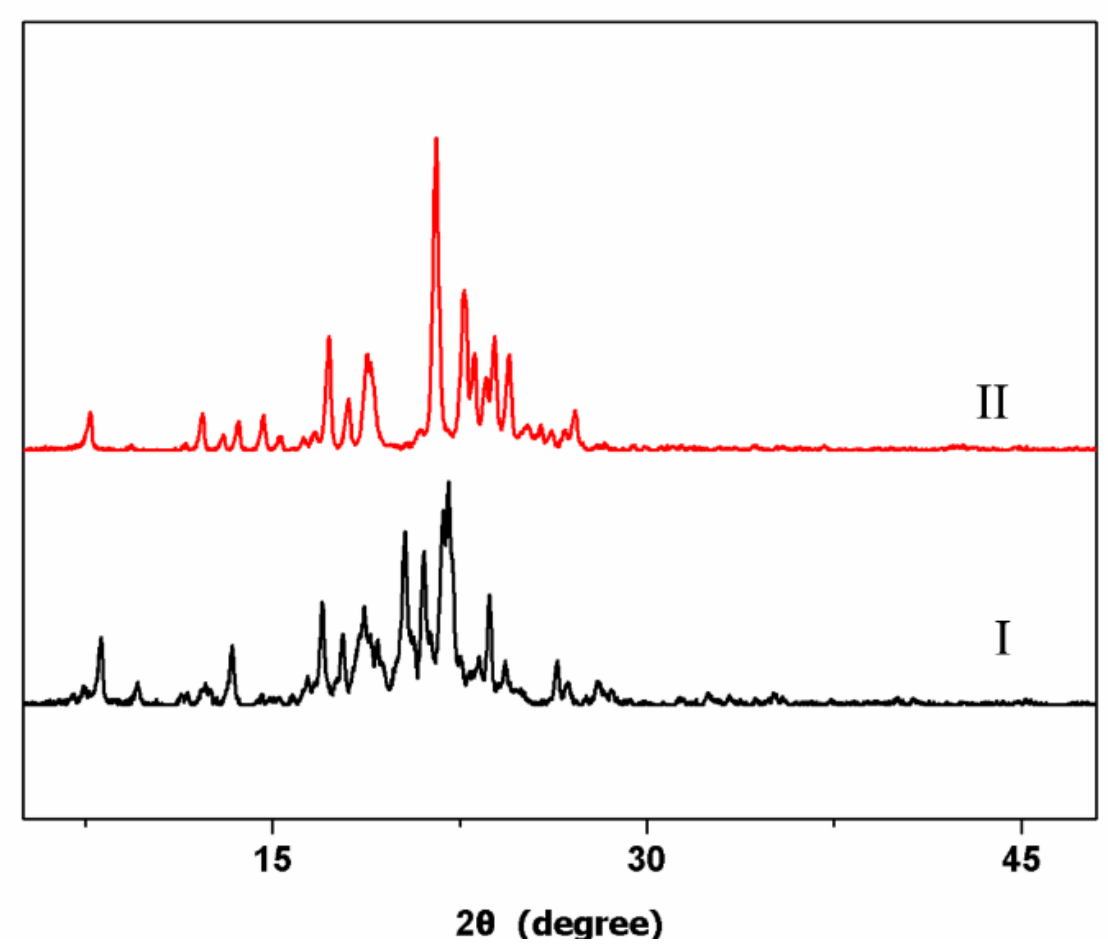

Figure S21. Powder X-ray diffraction patterns of BPy-Cage (I), BPy-Cage after release of iodine (II).

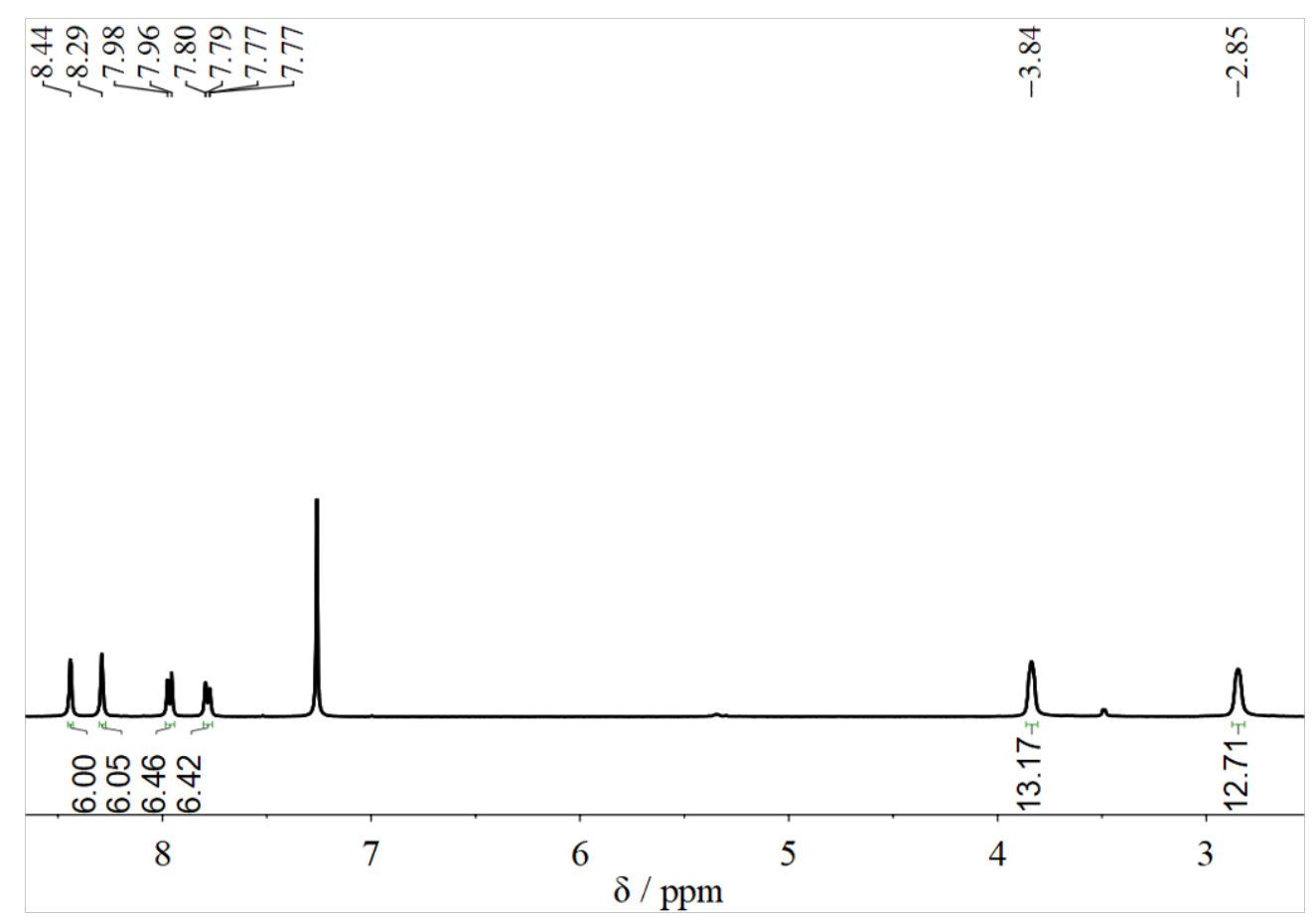

Figure S22. ${ }^{1} \mathrm{H}$ NMR spectrum $\left(400 \mathrm{MHz}, 298 \mathrm{~K}, \mathrm{CDCl}_{3}\right)$ of recycled BPy-Cage.

The recycled BPy-Cage solid was tested for its adsorptive capability. Here, $50 \mathrm{mg}$ BPyCage solid was added to an $\mathrm{I}_{2} / \mathrm{n}$-hexane solution $(2.50 \mathrm{mM}, 5 \mathrm{~mL})$. When the colour of the solution changed to colourless, it was subject to filtration. The resulting brown solid was treated 
with DMF until all the $\mathrm{I}_{2}$ was released as judged from spectroscopic analysis. Then the recycled BPy-Cage solid was added to $I_{2} /$ hexane solution again. After four cycles, the recycled BPyCage solid showed similar $\mathrm{I}_{2}$ capture performance in solution.

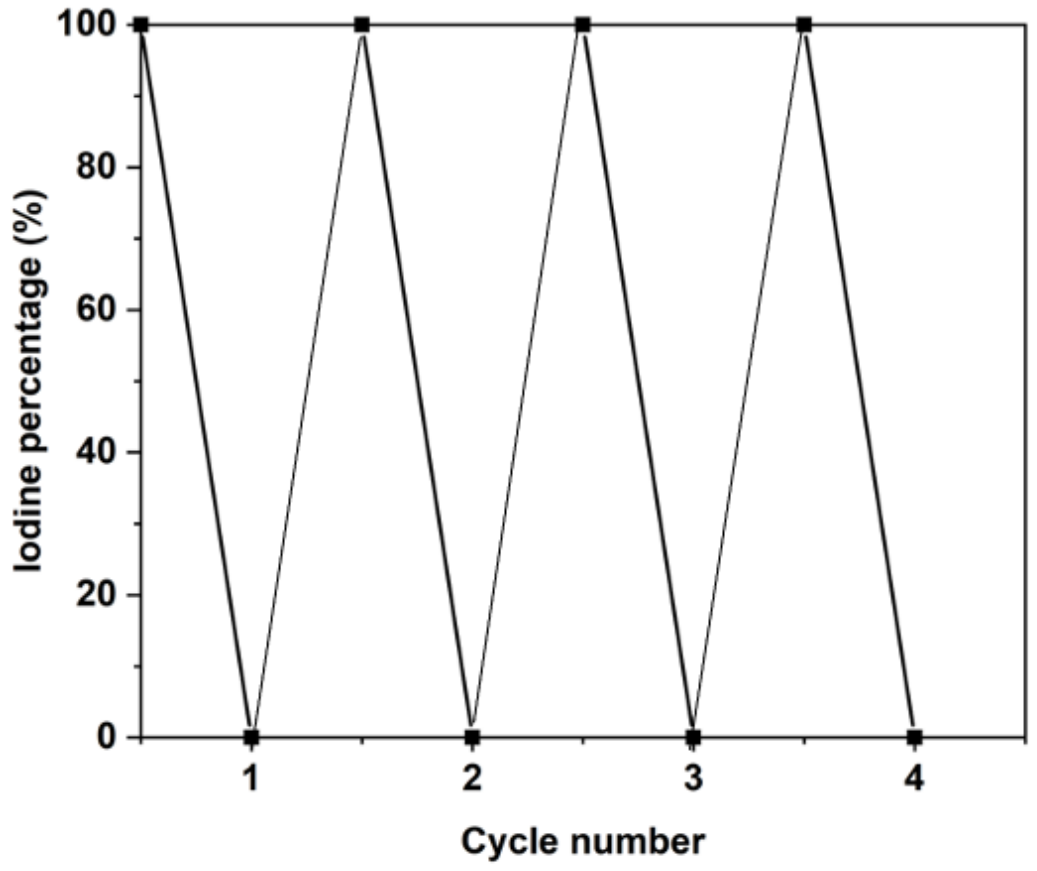

Figure S23. Tests of the reusability of BPy-Cage as an iodine capture material.

\section{Studies of BPy-Cage-Modulated Acylation}

Scheme S4. Synthesis of $p$-nitrobenzyl acetate under differing conditions

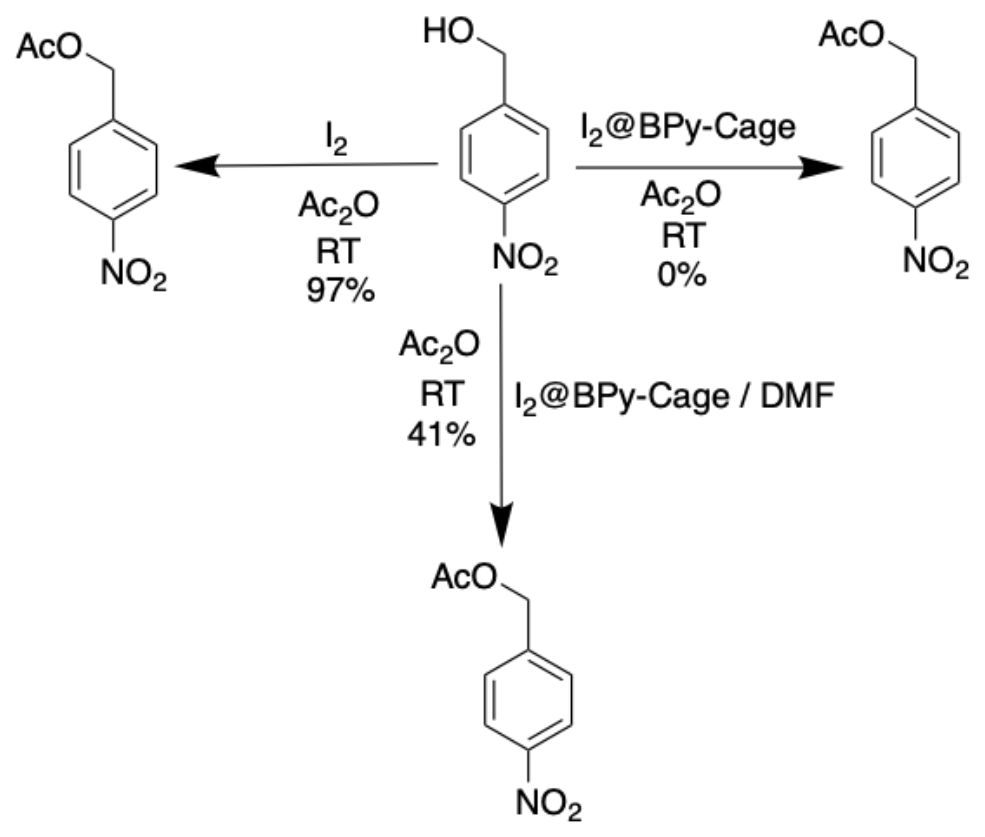


10. Iodine Adsorption Capacities of Various Porous Adsorbents.

Table S4. Iodine adsorption capacities of various porous adsorbents.

\begin{tabular}{|c|c|c|c|c|c|c|}
\hline Adsorbent & Catogery & $\begin{array}{r}\mathrm{T} \\
\left({ }^{\circ} \mathrm{C}\right)\end{array}$ & $\begin{array}{c}S_{\text {BET }} \\
\left(\mathrm{m}^{2}\right. \\
\left.\mathrm{g}^{-1}\right)\end{array}$ & $\begin{array}{c}\text { Bala } \\
\text { nce time } \\
\text { (h) }\end{array}$ & $\begin{array}{r}\text { Iodin } \\
\text { e capacity } \\
\left(\mathrm{g} \mathrm{g} \mathrm{g}^{-1}\right)\end{array}$ & Ref. \\
\hline BPy-Cage & $\begin{array}{l}\text { Nonporous } \\
\text { cage }\end{array}$ & 70 & 1.8 & & 3.23 & $\begin{array}{l}\text { This } \\
\text { work }\end{array}$ \\
\hline EtP6 $\beta$ & $\begin{array}{l}\text { Molecular } \\
\text { crystal }\end{array}$ & 85 & - & 2 & 0.200 & 1 \\
\hline ВТРОС & POC & 75 & 605 & 14 & 3.210 & 2 \\
\hline $\mathrm{CC} 3$ & POC & 20 & - & 350 & 0.450 & 3 \\
\hline Cage-1 & $\begin{array}{l}\text { Metal organic } \\
\text { coordination } \\
\text { cage }\end{array}$ & 75 & 137 & 36 & 1.420 & 4 \\
\hline TJNU-201 & $\mathrm{COF}$ & 77 & 2150 & 96 & 5.625 & 5 \\
\hline HCOF-1 & $\mathrm{HOF}$ & 75 & - & - & 2.900 & 6 \\
\hline $\begin{array}{l}\text { TPT-TAPB- } \\
\text { COF }\end{array}$ & $\mathrm{COF}$ & 75 & 957 & 12 & 2.250 & 7 \\
\hline CMPN-3 & $\mathrm{COF}$ & 70 & 1368 & 2.5 & 2.080 & 8 \\
\hline $\begin{array}{l}\text { MFM- } \\
300(\mathrm{Sc})\end{array}$ & MOF & 70 & 1250 & 10 & 1.540 & 9 \\
\hline $\begin{array}{l}\mathrm{UPC}-158- \\
\mathrm{HCl}\end{array}$ & MOF & 70 & 2289 & 20 & 2.920 & 10 \\
\hline
\end{tabular}


11. Iodine Adsorption Capacities of 2,2'-BPy-DA

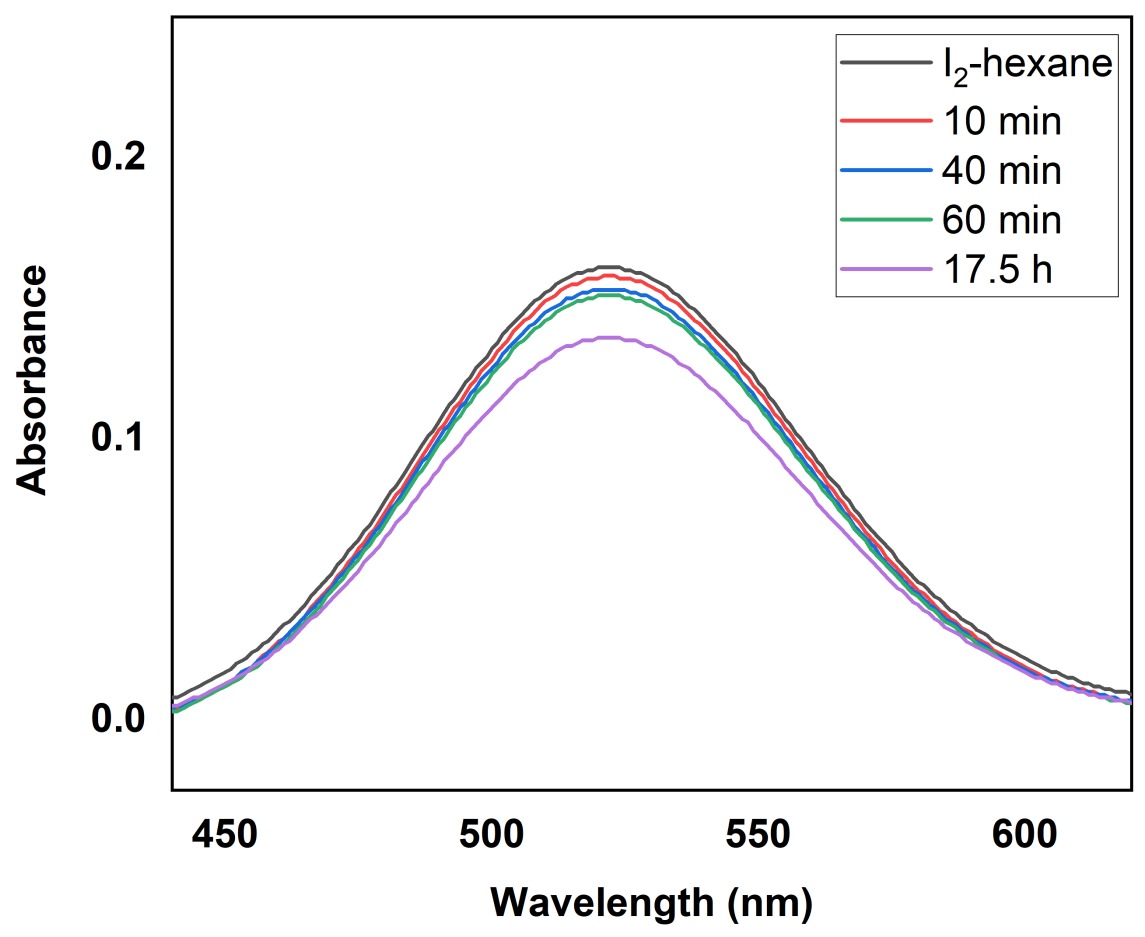

Figure S24. Time-dependent UV/vis absorption spectra of an iodine/n-hexane solution recorded upon addition of 2,2'-BPy-DA.

(b)

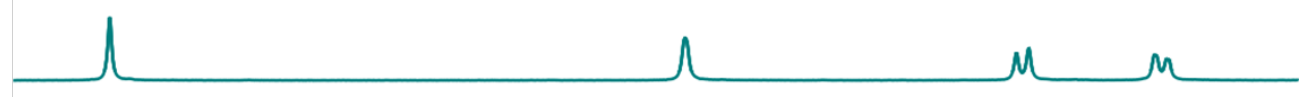

(a)

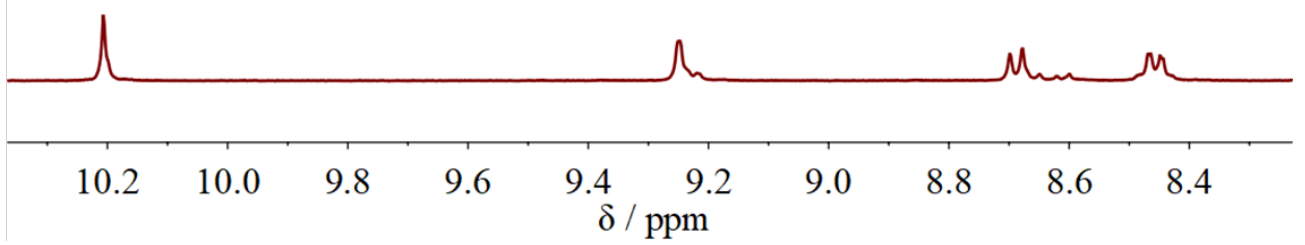

Figure S25. ${ }^{1} \mathrm{H}$ NMR spectrum (400 MHz, DMSO- $d_{6}, 298 \mathrm{~K}$ ) of (a) 2,2'-BPy-DA and (b) 2,2'BPy-DA + 2 equiv $I_{2}$. 
12. Studies of Bromine Capture from n-Hexane Solution

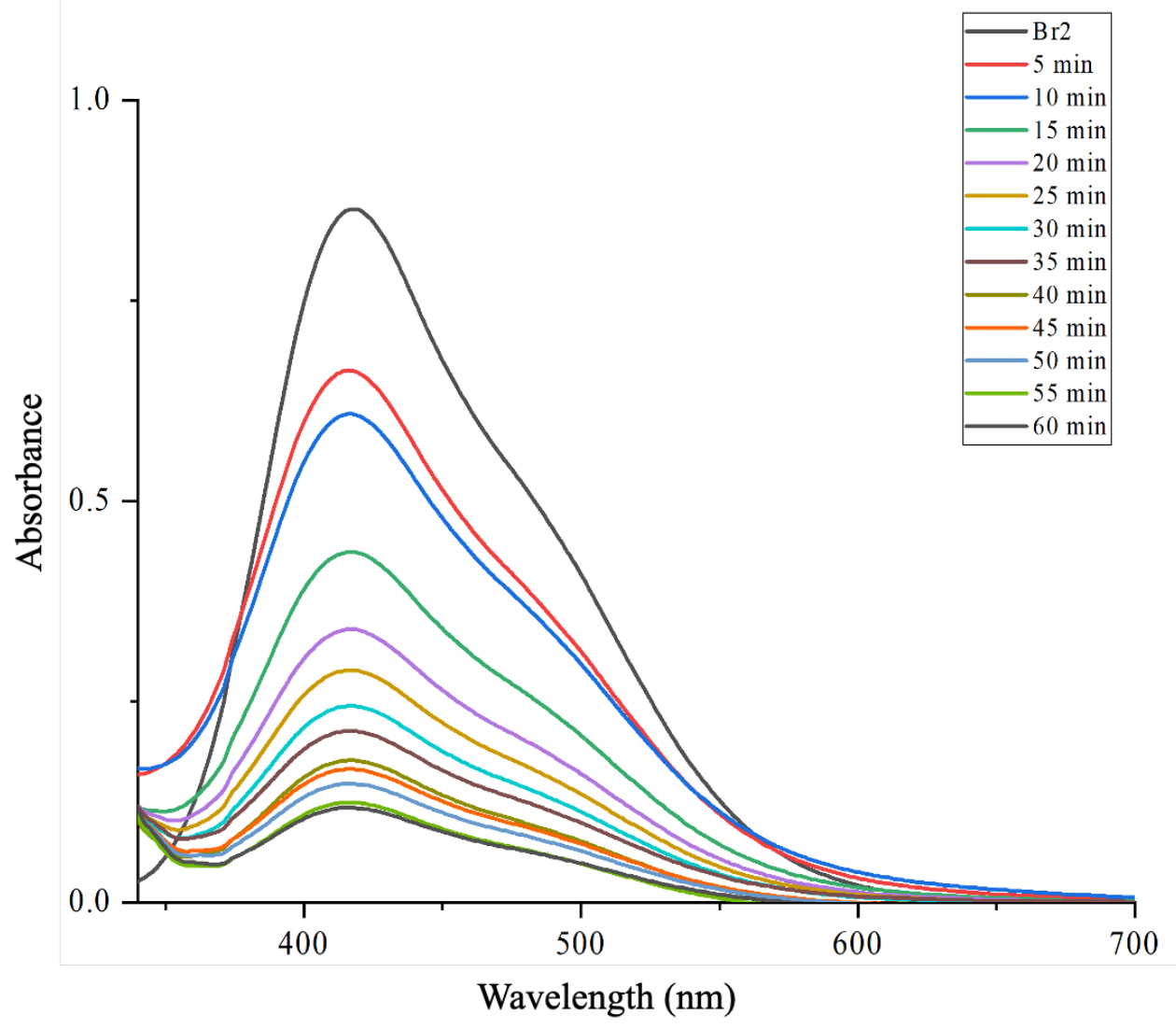

Figure S26. Time-dependent UV-Vis absorption spectra of a bromine n-hexane solution (4.8 $\mathrm{mM}$ ) recorded upon the addition of BPy-Cage. 


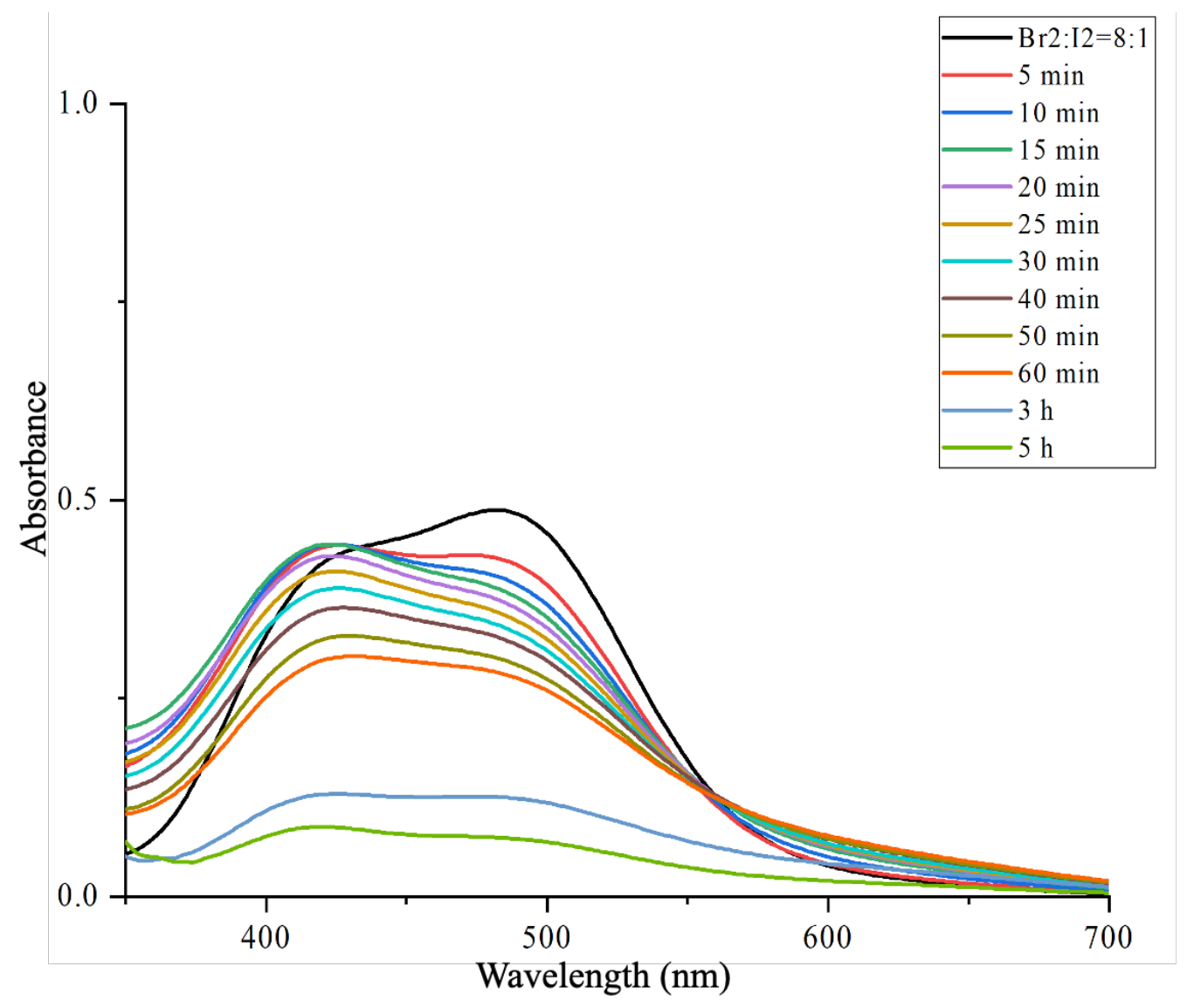

Figure S27. Time-dependent UV-vis absorption spectra of a bromine + iodine n-hexane solution $\left(\left[\mathrm{Br}_{2}\right]=4.0 \mathrm{mM} ;\left[\mathrm{I}_{2}\right]=0.5 \mathrm{mM}\right)$ recorded upon the addition of BPy-Cage.

\section{Supplemental References}

1. Jie, K.; Zhou, Y.; Li, E.; Li, Z.; Zhao, R.; Huang, F. J. Am. Chem. Soc. 2017, 139, 15320-15323.

2. Liu, C.; Li, W.; Liu, Y.; Wang, H.; Yu, B.; Bao, Z.; Jiang, J. Chem. Eng. J. 2022, 428, 131129.

3. Hasell, T.; Schmidtmann, M.; Cooper, A. I. J. Am. Chem. Soc. 2011, 133, 14920-14923.

4. Pei, W.-Y.; Yang, J.; Wu, H.; Zhou, W.; Yang, Y.-W.; Ma, J.-F. ChemComm. 2020, 56, 24912494.

5. Li, J.; Zhang, H.; Zhang, L.; Wang, K.; Wang, Z.; Liu, G.; Zhao, Y.; Zeng, Y. J. Mater. Chem. A 2020, 8, 9523-9527.

6. Lin, Y.; Jiang, X.; Kim, S. T. S.; Alahakoon, B.; Hou, X.; Zhang, Z.; Thompson, C. M.; Smaldone, R. A.; Ke, C. J. Am. Chem. Soc. 2017, 139, 7172-7175.

7. Chen, Y.; Sun, H.; Yang, R.; Wang, T.; Pei, C.; Xiang, Z.; Zhu, Z.; Liang, W.; Li, A.; Deng, W. J. Mater. Chem. A 2015, 3, 87-91.

8. Li, Y.; Chen, W.; Hao, W.; Li, Y.; Chen, L. ACS Appl. Nano Mater. 2018, 1, 4756-4761.

9. Zhang, X.; Silva, I. da; Godfrey, H. G. W.; Callear, S. K.; Sapchenko, S. A.; Cheng, Y.; Vitórica-Yrezábal, I.; Frogley, M. D.; Cinque, G.; Tang, C. C.; Giacobbe, C.; Dejoie, C.; Rudić, S.; Ramirez-Cuesta, A. J.; Denecke, M. A.; Yang, S.; Schröder, M. J. Am. Chem. Soc. 2017, 139, 16289-16296.

10. Guo, B.; Li, F.; Wang, C.; Zhang, L.; Sun, D. J. Mater. Chem. A, 2019 7, 13173-13179. 